\title{
Dimension transcendence and anomalous charge transport in magnets with moving multiple- $Q$ spin textures
}

\author{
Ying Su $\odot,{ }^{1}$ Satoru Hayami $\odot,{ }^{2}$ and Shi-Zeng Lin $\odot^{1}$ \\ ${ }^{1}$ Theoretical Division, T-4 and CNLS, Los Alamos National Laboratory, Los Alamos, New Mexico 87545, USA \\ ${ }^{2}$ Department of Physics, Hokkaido University, Sapporo 060-0810, Japan
}

(Received 10 April 2019; revised manuscript received 30 April 2019; accepted 15 January 2020; published 14 February 2020)

\begin{abstract}
Multiple- $Q$ spin textures, such as magnetic bubble and skyrmion lattices, have been observed in a large family of magnets. These spin textures can be driven into motion by external stimuli. The motion of spin textures affects the electronic states. Here we show that to correctly describe the electronic dynamics, the momentum space needs to be transcended to higher dimensions by including the ancillary dimensions associated with phason modes of the translational motion of the spin textures. The electronic states have nontrivial topology characterized by the first and second Chern numbers in the high-dimensional hybrid momentum space. This gives rise to an anomalous electric charge transport due to the motion of spin textures. By deforming the spin textures, a nonlinear response associated with the second Chern number can be induced and results in the piezoelectricity. The charge transport is derived from the semiclassical equation of motion of electrons that depends on the Berry curvature in the hybrid momentum space. Our results suggest that the motion of multiple- $Q$ spin textures has significant effects on the electronic dynamics and provides a platform to explore high-dimensional topological physics.
\end{abstract}

DOI: 10.1103/PhysRevResearch.2.013160

\section{INTRODUCTION}

Spatially localized spin textures with nontrivial topology, such as magnetic bubbles and skyrmions, have been observed in several families of material systems, including both metals and insulators, and heterostructures [1-4]. These spin textures can form a lattice that is manifested by the spin structure factor peaks associated with multiple- $Q$ vectors of the spin texture [1]. For this reason, these crystallized spin textures are also named as multiple- $Q$ spin textures. The spin textures respond to various external stimuli, including electric current [5-10], electric field [11,12], magnetic field [13,14], thermal gradient [15-17], and strain [18-20], which renders them as very promising entities for applications [21-23].

The static spin texture has significant effects on electronic wave functions and affects the dynamics of conduction electrons [24,25]. For instance, when electrons interact with a static skyrmion, they experience an effective magnetic field produced by the noncoplanar spin texture. As a consequence, there is a topological Hall effect, which has been observed experimentally [26-28]. The noncoplanar spin texture can open an energy gap in the electronic spectrum and stabilize a Chern insulator [25,29-31]. On the other hand, the spin texture can be driven into motion and the moving spin texture is expected to affect the electronic dynamics. In ferromagnetic metals,

Published by the American Physical Society under the terms of the Creative Commons Attribution 4.0 International license. Further distribution of this work must maintain attribution to the author(s) and the published article's title, journal citation, and DOI. the interaction between conduction electrons and moving spin textures can generate an emergent electric field that results in a spin motive force [7,32-34]. Nevertheless, how it works for a gapped system is not well understood.

In this paper, we reveal that the description of the dynamics of conduction electrons coupled to the moving multiple- $Q$ spin textures requires a transcendent high-dimensional momentum space. The high-dimensional momentum space is spanned by the physical dimensions and ancillary dimensions associated with phason modes of the translational motion of spin textures. The electronic states can have nontrivial topology characterized by the first and second Chern numbers in the hybrid momentum space when the energy spectrum is gapped. Due to the nontrivial topology, there is an anomalous charge transport in magnets with moving multiple- $Q$ spin textures. To explain the anomalous charge transport, we develop a general semiclassical theory to study the response of electronic states to the motion and deformation of spin textures. Our results demonstrate that the motion of multiple$Q$ spin textures has profound consequences on the electronic wave functions and dynamics.

\section{MODEL AND DIMENSION TRANSCENDENCE}

The interaction between conduction electrons and spin textures can be described by the Hamiltonian

$$
\mathcal{H}=-t \sum_{\langle i, j\rangle} c_{i}^{\dagger} c_{j}-J \sum_{i} c_{i}^{\dagger} \boldsymbol{S}_{i} \cdot \sigma c_{i}-B \sum_{i} c_{i}^{\dagger} \sigma_{z} c_{i},
$$

where $c_{i}=\left(c_{i, \uparrow}, c_{i, \downarrow}\right)^{\top}$ is the annihilation operator of conduction electrons with spin $\uparrow$ and $\downarrow$ at the $i$ th site, $\langle i, j\rangle$ denotes two nearest-neighbor sites on a two-dimensional (2D) 
square lattice, $\sigma$ represents the vector of Pauli matrices, and $\boldsymbol{S}_{i}$ encodes the spin texture. Here we assume that the spin texture $S_{i}$ is stabilized by other stronger interactions and is not affected by the conduction electrons. The spin configuration of the multiple- $Q$ spin textures can be generally described by the linear superposition of a set of periodic functions,

$$
\boldsymbol{S}_{i}=\sum_{\nu} G_{v}^{\mu}\left(\boldsymbol{Q}_{v} \cdot \boldsymbol{r}_{i}+\phi_{v}\right) \hat{\boldsymbol{e}}_{\mu},
$$

where $\mu=\{x, y, z\}$ denotes the real-space coordinates and $v=\{1,2, \ldots\}$ is the index for multiple $\boldsymbol{Q}$ vectors. Here the periodic function follows $G_{v}^{\mu}\left(\phi_{v}\right)=G_{v}^{\mu}\left(\phi_{\nu}+2 \pi\right)$, and the Einstein summation convention is employed hereafter. The translational motion of the spin texture is depicted by the time-dependent phase $\phi_{\nu}=\omega_{\nu} \tau$, where $\tau$ is time. Here we consider only the dominant $\boldsymbol{Q}_{v}$ vectors of the spin textures. The high harmonic terms, such as those at $2 \boldsymbol{Q}_{v}$, are usually weak enough to be neglected. In particular, the high harmonic contributions can be treated as weak perturbations, which do not affect the topological charge transport discussed below as long as the energy gap remains open.

The coupling of conduction electrons to multiple- $Q$ spin textures yields a magnetic superlattice. We denote the crystal momentum of the magnetic superlattice as $\boldsymbol{k}$. The Hamiltonian $H(\boldsymbol{k}, \boldsymbol{\phi})$ in the momentum space is a periodic function of both $\boldsymbol{k}$ and $\boldsymbol{\phi}=\left(\phi_{1}, \phi_{2}, \ldots\right)$ that configure a high-dimensional Hamiltonian manifold. Therefore, when the spin texture is moving, the electronic dynamics is depicted in the effective momentum space spanned by $\tilde{\boldsymbol{k}}=\boldsymbol{k} \oplus \boldsymbol{\phi}$, a hybridization of crystal momenta and phason modes associated with the translational motion of spin textures. Because the motion of spin textures is much slower than the electronic dynamics, the electronic states evolve adiabatically with the change of $\phi_{\nu}$. For instance, the typical velocity of skyrmion is of the order of $100 \mathrm{~m} / \mathrm{s}$ [35], while the group velocity of the electron can be of the order of $1 \times 10^{6} \mathrm{~m} / \mathrm{s}$, like in graphene. Therefore, we can treat $\phi_{\nu}$ as adiabatic variables and the dimension of the system is transcendent by considering $\phi_{v}$ as an extra dimension. If the system is topologically nontrivial in the high-dimensional space, one would expect a nontrivial charge transport driven by the motion of the spin texture as a consequence of topological pumping. As will be discussed below, this is indeed the case for the multiple- $Q$ spin textures studied in this work.

\section{NONLINEAR RESPONSE}

We use the semiclassical theory to describe the electronic dynamics in the hybrid high-dimensional space. The semiclassical equation of motion for an electronic wave packet with the dispersion $E_{n}(\tilde{\boldsymbol{k}})$ and center of mass $\boldsymbol{r}_{n}$ follows

$$
\dot{r}_{n}^{\mu}=\frac{\partial E_{n}(\tilde{\boldsymbol{k}})}{\hbar \partial k_{\mu}}-\dot{k}_{\gamma} \Omega_{n}^{\mu \gamma}(\tilde{\boldsymbol{k}})-\dot{\phi}_{\nu} \Omega_{n}^{\mu \nu}(\tilde{\boldsymbol{k}}),
$$

where $\Omega_{n}^{\mu \gamma}=i\left\langle\partial_{k_{\mu}} u_{n \tilde{k}} \mid \partial_{k_{\gamma}} u_{n \tilde{k}}\right\rangle-i\left\langle\partial_{k_{\gamma}} u_{n \tilde{k}} \mid \partial_{k_{\mu}} u_{n \tilde{k}}\right\rangle$ and $\Omega_{n}^{\mu \nu}=$ $i\left\langle\partial_{k_{\mu}} u_{n \tilde{k}} \mid \partial_{\phi_{v}} u_{n \tilde{k}}\right\rangle-i\left\langle\partial_{\phi_{v}} u_{n \tilde{k}} \mid \partial_{k_{\mu}} u_{n \tilde{k}}\right\rangle$ are the Berry curvatures defined in the real and hybrid momentum spaces, respectively. $\left|u_{n \tilde{k}}\right\rangle$ is the periodic part of the Bloch state $\left|\psi_{n \tilde{k}}\right\rangle$ and $\gamma$ is the index denoting real-space coordinates, that is summed over in Eq. (3). Here the first term is the group velocity, the second term describes the conventional anomalous velocity [36], and the last term is the anomalous velocity induced by the motion of spin textures that can be derived from the semiclassical theory (see Appendix A). In the absence of external electric field, $\dot{k}_{\gamma}=0$, the current density due to the translational motion of spin textures is

$$
j_{0}^{\mu}=e \sum_{n} \int \frac{d^{2} k}{4 \pi^{2}} f\left(E_{n}\right)\left(\frac{\partial E_{n}}{\hbar \partial k_{\mu}}-\omega_{\nu} \Omega_{n}^{\mu \nu}\right),
$$

where $\dot{\phi}_{v}=\omega_{v}$ and $f\left(E_{n}\right)$ is the Fermi distribution function. When the energy spectrum is gapped in the hybrid momentum space and the Fermi energy is in the gap for a magnetic insulator, the first term in Eq. (4) vanishes. The transported charge over one period of motion $\Delta \tau=2 \pi / \omega_{\nu}$ at zero temperature is

$$
\begin{aligned}
q_{0}^{\mu} & =-e N^{\mu} \sum_{E_{n} \leqslant E_{F}} \int d \tau \int \frac{d^{2} k}{4 \pi^{2}} \omega_{\nu} \Omega_{n}^{\mu \nu} \\
& =e N^{\mu} \operatorname{sign}\left(\omega_{\nu}\right) C_{1}^{\mu \nu}, \\
C_{1}^{\mu \nu} & =-\frac{1}{2 \pi} \sum_{E_{n} \leqslant E_{F}} \int d k_{\mu} d \phi_{\nu} \Omega_{n}^{\mu \nu},
\end{aligned}
$$

where we have used $\int \omega_{\nu} d \tau=\int d \phi_{\nu}$. Here, $C_{1}^{\mu \nu}$ is the first Chern number defined on the $k_{\mu} \phi_{\nu}$ plane and is independent of the other momenta if the energy gap remains open in the entire hybrid momentum space, and $N^{\mu}$ is the number of unit cells in the cross section perpendicular to the $\mu$ direction. Therefore, the translational motion of the spin textures can result in quantized charge transport in magnetic insulators with $C_{1}^{\mu \nu} \neq 0$, which is just the topological pumping.

The response function given by Eq. (4) becomes nonlinear when the dynamics of $\phi_{v}$ couples with $\dot{r}_{n}$. This can be achieved by deforming the spin texture, $\boldsymbol{Q}_{v} \rightarrow \boldsymbol{Q}_{v}+\boldsymbol{Q}_{v}^{\prime}$. Therefore, $\phi_{\nu}=\omega_{\nu} \tau+\boldsymbol{Q}_{v}^{\prime} \cdot \boldsymbol{r}_{n}$, and its dynamics follows

$$
\dot{\phi}_{\nu}=\omega_{v}+Q_{v \mu}^{\prime} \dot{r}_{n}^{\mu},
$$

which depends on the velocity of the wave packet. Substituting Eq. (7) into Eq. (3) and retaining the terms up to the second order in $\omega_{v}$ and $Q_{v \mu}^{\prime}$, we obtain

$$
\begin{aligned}
\dot{r}_{n}^{\mu}= & \frac{\partial E_{n}}{\hbar \partial k_{\mu}}-\omega_{\nu} \Omega_{n}^{\mu \nu} \\
& -\left(\frac{\partial E_{n}}{\hbar \partial k_{\gamma}}-\omega_{\delta} \Omega_{n}^{\gamma \delta}-\frac{\partial E_{n}}{\hbar \partial k_{\eta}} Q_{\delta \eta}^{\prime} \Omega_{n}^{\gamma \delta}\right) Q_{\nu \gamma}^{\prime} \Omega_{n}^{\mu \nu},
\end{aligned}
$$

where the indexes $\{\mu, \gamma, \eta\}$ denote real-space coordinates and $\{v, \delta\}$ label different $\boldsymbol{Q}$ vectors. Here the first two terms correspond to the linear response in Eq. (4), while the last term originated from the deformation of spin textures induces a nonlinear response,

$$
j^{\mu}=j_{0}^{\mu}+e \sum_{n} \int \frac{d^{2} k}{8 \pi^{2}} f\left(E_{n}\right) F_{n}^{\mu \nu \gamma \delta} \omega_{\nu} Q_{\gamma \delta}^{\prime},
$$

where $F_{n}^{\mu \nu \gamma \delta}=\Omega_{n}^{\mu \nu} \Omega_{n}^{\gamma \delta}+\Omega_{n}^{\mu \gamma} \Omega_{n}^{\delta \nu}+\Omega_{n}^{\mu \delta} \Omega_{n}^{\nu \gamma}$ (see Appendix B). In this case, the transported charge over $\Delta \tau$ at zero 
temperature is

$$
\begin{aligned}
q^{\mu} & =q_{0}^{\mu}+e L^{\mu} \sum_{E_{n} \leqslant E_{F}} \int d \tau \int \frac{d^{2} k}{8 \pi^{2}} F_{n}^{\mu \nu \gamma \delta} \omega_{\nu} Q_{\gamma \delta}^{\prime} \\
& \approx q_{0}^{\mu}+\frac{e L^{\mu} \operatorname{sign}\left(\omega_{\nu}\right) C_{2}^{\mu \nu \gamma \delta} Q_{\gamma \delta}^{\prime}}{4 \pi},
\end{aligned}
$$

where $L^{\mu}$ is the size of the cross section perpendicular to the $\mu$ direction and

$$
C_{2}^{\mu \nu \gamma \delta}=\frac{1}{4 \pi^{2}} \sum_{E_{n} \leqslant E_{F}} \int d k_{\mu} d \phi_{\nu} d k_{\gamma} d \phi_{\delta} F_{n}^{\mu \nu \gamma \delta}
$$

is the second Chern number [37] defined on the $k_{\mu} \phi_{\nu} k_{\gamma} \phi_{\delta}$ hypersurface of the hybrid momentum space. When the period of the multiple- $Q$ spin texture is much longer than the atomic lattice parameter, the Berry curvatures are weakly dispersive as a function of $\phi_{\delta}$. This allows us to approximate $F_{n}^{\mu \nu \gamma \delta} \approx$ $\int d \phi_{\delta} F_{n}^{\mu \nu \gamma \delta} / 2 \pi$ in Eq. (10).

In our definition, both $C_{1}^{\mu \nu}$ and $C_{2}^{\mu \nu \gamma \delta}$ are antisymmetric under the permutation of the indices because $\Omega_{n}^{\mu \nu}=-\Omega_{n}^{v \mu}$. Equation (10) demonstrates that in addition to the contribution from the first Chern number, the charge transport has the contribution from the second Chern number when the spin texture is deformed.

To substantiate the anomalous charge transport due to the translational motion of the spin textures from the semiclassical analysis above, we study the charge transport in magnets with several typical multiple- $Q$ spin textures obtained from the Monte Carlo simulation (see Appendix C). Here we consider three kinds of spin textures: double- $Q$ and triple$Q$ collinear magnetic bubble lattices where spins are nearly parallel or antiparallel due to the strong easy-axis anisotropy, and a triple- $Q$ skyrmion lattice, as shown in Figs. 1(a)-1(c), respectively. For conduction electrons hopping on a square lattice (whose lattice constant is set to unity), the $\boldsymbol{Q}_{v}$ vectors for the double- $Q$ magnetic bubble lattice are $Q_{1}=(2 \pi / 5,0)$ and $\boldsymbol{Q}_{2}=(0,2 \pi / 5)$, while for the triple- $Q$ magnetic bubble and skyrmion lattices, they are $\boldsymbol{Q}_{1}=(-2 \pi / 5,2 \sqrt{3} \pi / 5), \boldsymbol{Q}_{2}=$ $(-2 \pi / 5,-2 \sqrt{3} \pi / 5)$, and $\boldsymbol{Q}_{3}=(4 \pi / 5,0)$. Here we consider the spin textures commensurate with the lattice to simplify the numerical calculation. For the triple- $Q$ states, because $\sum_{v=1}^{3} \phi_{v}=0$ for a translational motion, only two of $\phi_{v}$ are independent of each other.

\section{TOPOLOGICAL PROPERTY}

To identify the topology of electronic states in magnets with the multiple- $Q$ spin textures, we first study their electronic spectra in the hybrid momentum space. For the double$Q$ magnetic bubble lattice, the translational motion of the spin texture is parameterized by $\phi_{\nu}=\omega_{\nu} \tau$. The energy spectrum as a function of $\phi_{2}$ (with $\phi_{1}=0$ ) is shown in Fig. 1(d) for the double- $Q$ spin texture with $J=1.5 t$ and $B=0$. Here we use the periodic boundary condition along the $x$ direction and the open boundary condition along the $y$ direction. Apparently, there are topological edge states in the bulk energy gaps, indicating that the system is topologically nontrivial. For the triple- $Q$ spin textures, to make them commensurate with the lattice, we compress the lattice constant along the $y$ direc-
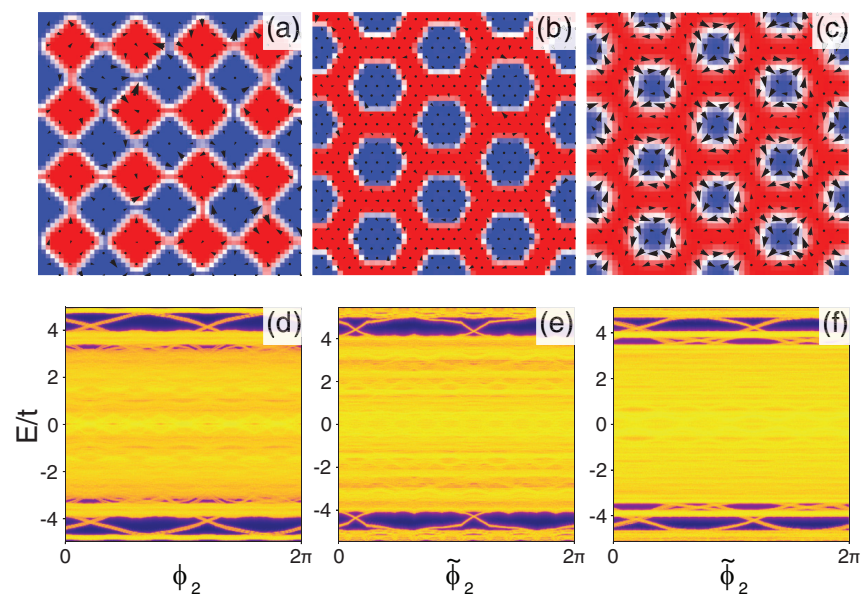

FIG. 1. Spin textures obtained from the Monte Carlo simulation (see Appendix C). (a) The double- $Q$ collinear magnetic bubble lattice. (b) The triple- $Q$ collinear magnetic bubble lattice. (c) The triple- $Q$ skyrmion lattice. Here the arrows represent the in-plane components, and the background color (with red for positive and blue for negative) stands for the $z$ component of local spins. (d)-(f) Electronic spectral functions as a function of $\phi_{2}\left(\tilde{\phi}_{2}\right)$ for conduction electrons coupled to the three spin textures (a)-(c), respectively. Here the purple (yellow) denotes the low (high) density of states.

tion to $1 / \sqrt{3}$. In this case, because $\sum_{\nu=1}^{3} \phi_{v}=0$, we define $\tilde{\phi}_{1}=-\left(\phi_{1}+\phi_{2}\right) / 2=\phi_{3} / 2$ and $\tilde{\phi}_{2}=\left(\phi_{1}-\phi_{2}\right) / 2$ that are independent. The change of $\tilde{\phi}_{1}$ and $\tilde{\phi}_{2}$ corresponds to the shift of triple- $Q$ spin textures along the $x$ and $y$ directions, respectively. Thus we can parametrize the translational motion of the triple- $Q$ spin textures by $\tilde{\phi}_{v}=\omega_{v} \tau$. The energy spectra as a function of $\tilde{\phi}_{2}$ (with $\tilde{\phi}_{1}=0$ ) for the triple- $Q$ magnetic bubble and skyrmion lattices with $J=1.5 t$ and $B=-1.2 t$ are displayed in Figs. 1(e) and 1(f), respectively. The topological edge states and bulk energy gaps persist for the triple- $Q$ spin textures.

To characterize the nontrivial band topology, we calculate the Chern numbers of the system with an efficient algorithm by using the U(1) link variable [38,39]. For commensurate spin textures, the system has translational symmetry and we can describe it in a high-dimensional hybrid momentum space spanned by the generalized crystal momentum $\tilde{\boldsymbol{k}}=\left(k_{x}, k_{y}, \phi_{1}, \phi_{2}\right)$ for the double- $Q$ spin texture and $\tilde{\boldsymbol{k}}=$ $\left(k_{x}, k_{y}, \tilde{\phi}_{1}, \tilde{\phi}_{2}\right)$ for the triple- $Q$ spin textures. Even if the spin texture is incommensurate with the lattice, $k_{x}$ and $k_{y}$ can still be introduced by using the twisted-boundary condition [40]. Consequently, the Chern numbers in Eqs. (6) and (11) are well defined on the compact manifold. As an example, we consider the Fermi energy in the bottom bulk energy gaps in Figs. 1(d)-1(f). In the 4D hybrid momentum space, there are six first Chern numbers: $C_{1}^{x 1}=C_{1}^{y 2}=2$ and $C_{1}^{x y}=C_{1}^{x 2}=$ $C_{1}^{y 1}=C_{1}^{12}=0$, and one second Chern number: $C_{2}^{x 1 y 2}=-2$ for the double magnetic bubble lattice. For the triple- $Q$ magnetic bubble and skyrmion lattices, they have the same Chern numbers: $C_{1}^{x 1}=C_{1}^{y 2}=2, C_{1}^{x y}=C_{1}^{x 2}=C_{1}^{y 1}=C_{1}^{12}=0$, and $C_{2}^{x 1 y 2}=-4$. Interestingly, our results demonstrate that an insulator that is trivial in the crystal momentum space can be topologically nontrivial in the hybrid momentum space. 

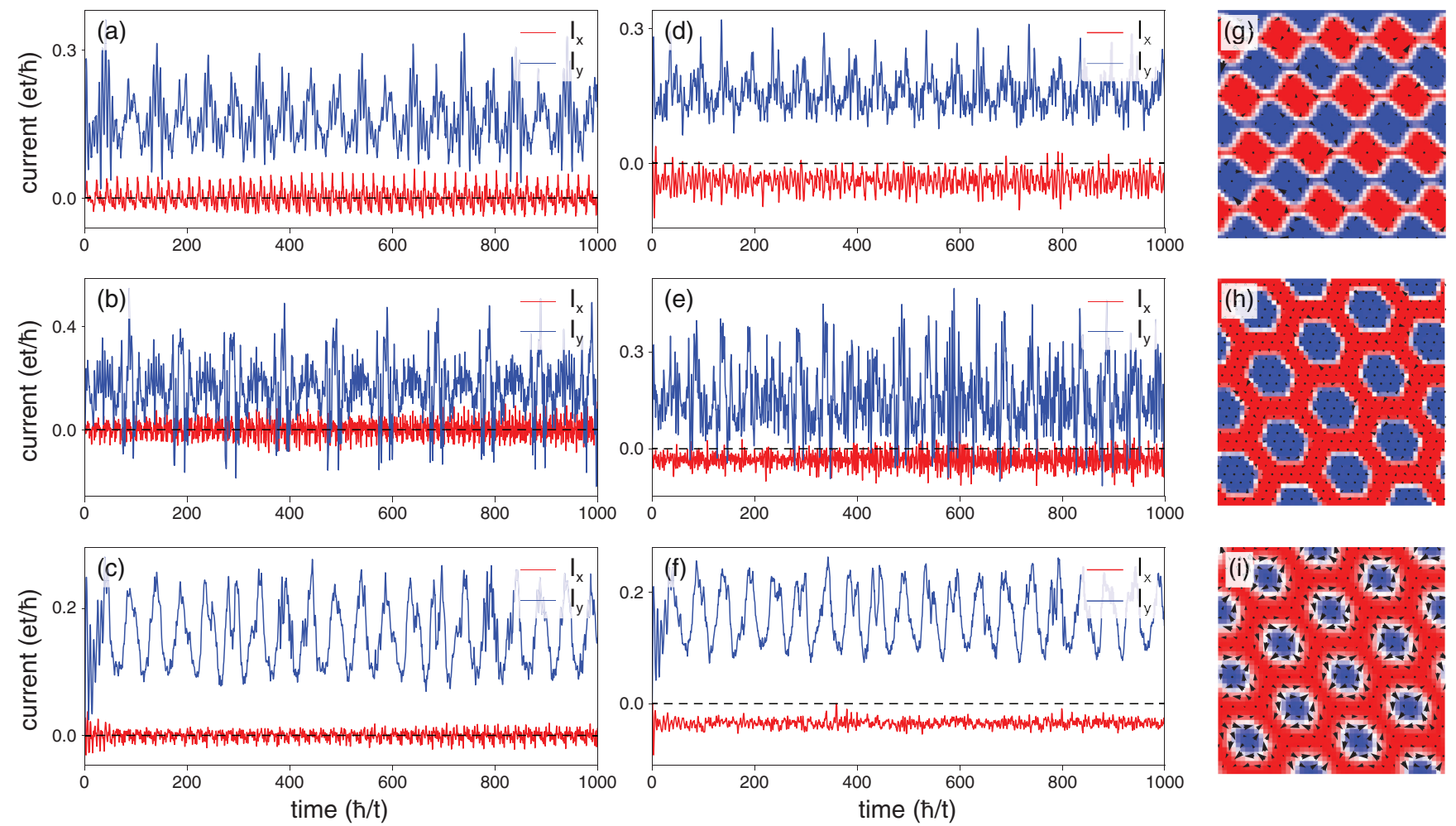

FIG. 2. Currents along the $x$ and $y$ directions induced by the translational motion of spin textures. (a) Currents for the double- $Q$ magnetic bubble lattice in Fig. 1(a); (b) currents for the triple- $Q$ magnetic bubble lattice in Fig. 1(b); and (c) currents for the triple- $Q$ skyrmion lattice in Fig. 1(c). (d)-(f) Currents for the three spin textures under a shear deformation. (g)-(i) Sheared spin textures are obtained by applying a shear strain on the spin textures in Figs. 1(a)-1(c).

To facilitate the understanding of nontrivial topology in the high-dimensional hybrid space, we show that the model can be mapped to a high-dimensional Hofstadter model for a simple collinear spin texture ansatz (see Appendix E). According to the mapping, the $\boldsymbol{Q}_{v}$ vector and the frequency $\omega_{v}$ of spin texture motion effectively play the role of magnetic and electric fields, respectively. Therefore, the high-dimensional nontrivial topology can be induced by the multiple- $Q$ spin textures and the motion of spin textures generates an electric current.

\section{ANOMALOUS CHARGE TRANSPORT}

The translational motion of spin textures can induce a quantized charge transport according to Eqs. (5) and (6). Here we consider the spin textures shifting along the $y$ direction on a $40 \times 40$ lattice (containing $8 \times 8$ supercells) with $\omega_{1}=0$ and $\omega_{2}=2 \pi t / 100 \hbar$, and we calculate the electric currents of the system by solving the time-dependent Schrödinger equation (see Appendix D). We focus on the Fermi energy in the bottom bulk energy gaps in Figs. 1(d)-1(f). For the double- $Q$ magnetic bubble lattice, the currents along the $x$ and $y$ directions over ten periods are shown in Fig. 2(a). By integrating the currents over time, the average transported charges in one period are $q^{x}=0.01 e$ and $q^{y}=15.95 e$. According to Eq. (5), $q^{x}=e N^{x} C^{x 2}=0$ and $q^{y}=e N^{y} C^{y 2}=16 e$. Apparently, the number obtained by simulation shows a good agreement with that obtained from the semiclassical equation. For the triple- $Q$ magnetic bubble lattice, we have $q^{x}=0.14 e$ and $q^{y}=16.04 e$ from the currents in Fig. 2(b), while for the triple- $Q$ skyrmion lattice, we have $q^{x}=0.00 e$ and $q^{y}=$ 15.96e from the currents in Fig. 2(c). The numerical results for the triple- $Q$ spin textures are also consistent with $q^{x}=0$ and $q^{y}=16 e$ obtained from Eq. (5).

The nonlinear response can be realized by deforming the spin texture as shown in Eqs. (7) and (9). For the double$Q$ magnetic bubble lattice, we deform the spin texture by setting $\phi_{1}=Q_{1}^{\prime} \cdot \boldsymbol{r}$ with $\boldsymbol{Q}_{1}^{\prime}=(0,2 \pi / 20)$. This induces a shear strain $\gamma_{x y}=-Q_{1 y}^{\prime} / Q_{1 x}=-0.25$ on the spin texture that results in $x \rightarrow x+\gamma_{x y} y$, as shown in Fig. 2(g). The currents due to the translational motion of the spin texture under shear strain are displayed in Fig. 2(d). In this case, the transported charges in one period are $q^{x}=-3.92 e$ and $q^{y}=$ $15.89 e$ from the currents in Fig. 2(d). Meanwhile, according to Eq. (10), we have $q^{x}=e L^{x} C_{2}^{x 21 y} Q_{1 y}^{\prime} / 2 \pi=-4 e$ where $C_{2}^{x 21 y}=C_{2}^{x 1 y 2}=-2$, and $q^{y}=16 e$ as before. The results from numerical simulation and semiclassical analysis are consistent with each other. Therefore, we demonstrate that besides the longitudinal charge transport due to the linear response, there is a transverse charge transport caused by the nonlinear response of the deformed spin texture. Moreover, one can probe the Chern numbers experimentally from the quantized charge transport. For the triple- $Q$ spin textures, the shear strain can also be induced by taking $\tilde{\phi}_{1}=\boldsymbol{Q}_{1}^{\prime} \cdot \boldsymbol{r}$, where we set $\boldsymbol{Q}_{1}^{\prime}=(0,2 \sqrt{3} \pi / 40)$ and $\gamma_{x y}=Q_{1 y}^{\prime} / Q_{1 x}=-0.125 \sqrt{3}$ in Figs. 2(h) and 2(i). The currents of the triple- $Q$ magnetic bubble lattice are shown in Fig. 2(e) and the transported charges in one period of motion are $q^{x}=-3.43 e$ and $q^{y}=15.60 e$. 
The currents of the triple- $Q$ skyrmion lattice are shown in Fig. 2(f) and the transported charges are $q^{x}=-3.48 e$ and $q^{y}=15.92 e$. Equation (10) yields $q^{x}=-4 e$ and $q^{y}=16 e$ for the triple- $Q$ spin textures. In this case, the numerical results are less quantized. One possible reason is due to the fact that the spin textures from Monte Carlo simulation are not exactly periodic. As a comparison, we also calculate the current for the double- $Q$ and triple- $Q$ spin texture ansatz described by the linear superposition of cosine functions. In this case, the transported charge is perfectly quantized (see Appendix E).

When the deformed spin texture is commensurate with the lattice, one may wonder whether the anomalous charge transport can be fully characterized by the first Chern numbers of the deformed system. To answer this question, we take the deformed double- $Q$ magnetic bubble lattice studied above as an example. Under the deformation induced by $\boldsymbol{Q}_{1}^{\prime}=$ $(0,2 \pi / 20)$, the new unit cell is enlarged to contain $5 \times 20$ lattice sites (fourth larger than the unit cell for the undeformed spin texture that is $5 \times 5$ ). Therefore, on the $40 \times 40$ lattice, $N_{x}=2$ and $N_{y}=8$. Because the lattice constant along the $y$ direction is increased by four times, the time period of motion of the spin texture is also increased by four times (since $\omega_{2}$ is fixed). By performing exactly the same calculation, we find that the relevant first Chern numbers of the deformed system become $C_{1}^{x 2}=-8$ and $C_{1}^{y 2}=8$. Plugging these into Eq. (5), we get $q^{x}=-16 e$ and $q^{y}=64 e$ in one period of motion. To compare with the previous results, we need to divide the new $q^{x}$ and $q^{y}$ by 4 to count the transported charges in the same period of time as the undeformed case. Thus, the two independent approaches yield the same result. As shown in this example, a small deformation of spin textures can increase the size of the unit cell significantly. With the periodic modulation extended to a larger length scale, there are more energy bands due to the band folding, accompanying complicated band-gap closing and opening in this process, where the topological phase transitions are possible. Therefore, without employing the nonlinear response function associated with the second Chern number, one needs to calculate different first Chern numbers for different deformation to understand the charge transport. This calculation turns out to be impractical for small deformation that results in huge unit cells. In this sense, the nonlinear response theory can make life much easier since one only needs to know the first and second Chern numbers of the undeformed system and these Chern numbers are usually relatively easy to calculate.

\section{LINEAR PIEZOELECTRICITY}

As we have shown above, the deformation of spin textures is essential to generate the nonlinear response. The deformation of magnetic skyrmion lattices has been realized in a strained crystal [20] or by applying an electric field [41]. Very recently, the deformation of the moving magnetic skyrmion lattice under electric current flow has also been observed [42]. According to Eq. (9), the current depends linearly on the deformed $\boldsymbol{Q}_{v}^{\prime}$ vector, i.e., $j^{\mu} \propto Q_{\gamma \delta}^{\prime}$. For instance, when the spin texture is deformed by an effective shear strain $\gamma_{x y} \propto Q_{1 y}^{\prime}$ in our numerical simulation above, the linear piezoelectric current is $j^{x} \propto \gamma_{x y}$. To verify the linear piezoelectricity, we calculate $q^{x}$ as a function of $\gamma_{x y}$. The numerical results are
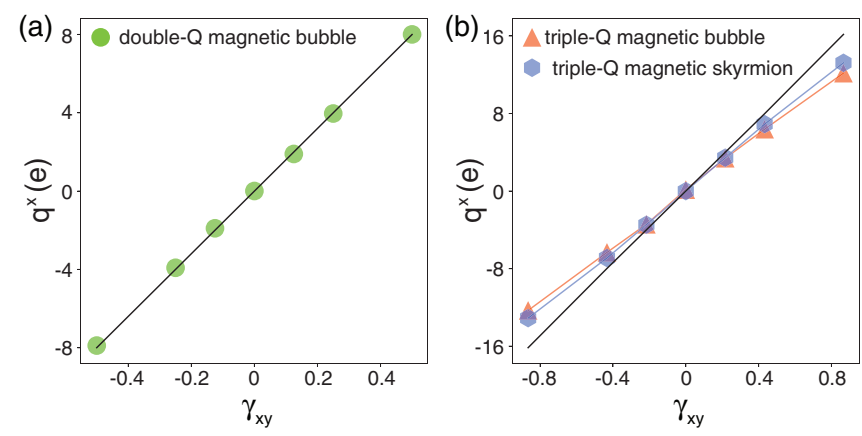

FIG. 3. Linear piezoelectricity induced by the deformation of spin textures. (a) $q^{x}$ as a function of the deformation $\gamma_{x y}$ for the double- $Q$ magnetic bubble lattice, and (b) for the triple- $Q$ magnetic bubble and skyrmion lattices. The black lines are from the response function given by Eq. (10).

displayed in Fig. 3. For the finite system size $(40 \times 40$ lattice sites) considered in our numerical calculations, the shear strain is limited to a few discrete values in order to retain the periodic boundary condition. For much larger systems, the shear strain can be tuned continuously. We show $q^{x}$ as a function of $\gamma_{x y}$ for the double- $Q$ magnetic bubble lattice in Fig. 3(a), and for the triple- $Q$ magnetic bubble and skyrmion lattices in Fig. 3(b). Here the symbols are from the numerical calculation and the black lines are from Eq. (10). Apparently, the $q^{x}$ for the double- $Q$ spin texture collapses on the black line and shows a good linear dependence on $\gamma_{x y}$. The $q^{x}$ for the triple- $Q$ spin textures are also linear in $\gamma_{x y}$, but deviate from the black line due to poor quantization of charge transport as reasoned above. Therefore, the linear piezoelectricity is verified numerically and works for moderate strains that preserve the intrinsic topology. Our results suggest a mechanism of piezoelectricity as a consequence of the nontrivial topology.

\section{DISCUSSION}

The nonlinear response due to the translational motion and deformation of the spin textures is associated with the second Chern number. The quantized charge transport requires the Fermi energy in the bulk energy gap. When this is not the case, Eq. (9) is still valid but the transported charge is not quantized. Therefore, the anomalous charge transport generated by the motion of spin textures occurs both in insulating and metallic magnets. In our system, the electronic dynamics is governed by the nontrivial topology of electronic structure in the hybrid momentum space spanned by the crystal momentum and phason mode of the spin texture. Our predictions can be tested in B20 chiral magnets [1,2], $f$-electron materials [43,44], and magnetic heterostructures [45-47], where multiple- $Q$ spin textures have been observed in experiments. The quantized charge transport can be possibly realized in a heterostructure with the multiple- $Q$ spin texture formed in a magnetic insulator layer or at the interface through the proximity effect [48-50]. In materials, the multiple- $Q$ spin textures are pinned by defects. To drive the spin texture into motion, a large driving force is needed in order to overcome the pinning energy. The pinning-depinning transition provides a clear way to distinguish the anomalous charge transport from other 
contributions. The motion of the noncoplanar spin texture can also induce a charge current due to the emergent electric field originated from the interaction between conduction electrons and the spin texture [7,32-34]. However, this current depends on the quasiparticle scattering lifetime, which is different from the topological charge transport caused by the motion of spin texture. For coplanar or collinear spin textures, the current due to the emergent electric field is absent, while the topological charge transport persists.

In summary, we reveal that the motion of spin textures has significant effects on the dynamics of conduction electrons. The semiclassical equation of motion of the electronic wave packet is described in a high-dimensional hybrid momentum space. The hybrid momentum space is constituted of the physical dimensions and ancillary dimensions associated with the phason modes of the translational motion of the spin textures. The electronic states can be topologically nontrivial when the energy spectrum is gapped, and the nontrivial topology is characterized by the first and second Chern numbers in the hybrid momentum space. The nontrivial topology results in an anomalous charge transport. The charge transport is quantized and can be used to extract the Chern numbers experimentally. Our theory presents a mechanism for electriccurrent generation induced by the motion of multiple- $Q$ spin texture in magnetic insulators. Our results can be extended to 3D magnets with multiple- $Q$ spin textures where even higher dimensional topological physics can be realized.

\section{ACKNOWLEDGMENTS}

We would like to thank Joel Moore, Chih-Chun Chien, and Cristian Batista for insightful discussions. Computer resources for the numerical calculations were supported by the Institutional Computing Program at LANL. This work was carried out under the auspices of the US Department of Energy NNSA under Contract No. 89233218CNA000001 through the LDRD Program, and was supported by the Center for Nonlinear Studies at LANL (Y.S. and S.Z.L). S.H. is supported by the JSPS KAKENHI Grant No. JP18K13488.

\section{APPENDIX A: SEMICLASSICAL EQUATION OF MOTION}

Here we consider a very general model of free electrons coupled to a periodic modulation potential described by

$$
H_{0}=\frac{\boldsymbol{p}^{2}}{2 m}+\sum_{v} V_{v}\left(\boldsymbol{Q}_{v} \cdot \boldsymbol{r}\right),
$$

and we study how the electron dynamics is affected by the motion and deformation of the potential. The potential is the linear superposition of a set of periodic functions $V_{v}\left(\boldsymbol{Q}_{v} \cdot \boldsymbol{r}\right)$ with multiple modulating vector $\boldsymbol{Q}_{v}$. The perturbation can be induced by taking $V_{v}\left(\boldsymbol{Q}_{v} \cdot \boldsymbol{r}\right) \rightarrow V_{v}\left(\boldsymbol{Q}_{v} \cdot \boldsymbol{r}+\phi_{v}\right)$, where

$$
\phi_{v}(\boldsymbol{r}, \tau)=\omega_{v} \tau+\boldsymbol{Q}_{v}^{\prime} \cdot \boldsymbol{r} .
$$

Here the first term describes the shift of the potential and the second term describes the deformation of the potential. Apparently, the multiple- $Q$ spin texture model belongs to the category depicted by Eq. (A1). In the semiclassical approach, the electronic state is described by a wave packet that is highly localized at the center of mass $\boldsymbol{r}_{c}$ and $\boldsymbol{k}^{c}$ in the phase space. Due to the localized nature, when the perturbation is smooth compared to the spread of the wave packet, the approximate Hamiltonian for the wave packet can be obtained by linearizing the perturbation as $\phi_{v}(\boldsymbol{r}, \tau) \approx \phi_{v}\left(\boldsymbol{r}_{c}, \tau\right)$ [36]. The leading-order local Hamiltonian is

$$
H_{c}=\frac{\boldsymbol{p}^{2}}{2 m}+\sum_{v} V_{v}\left(\boldsymbol{Q}_{v} \cdot \boldsymbol{r}+\phi_{v}\left(\boldsymbol{r}_{c}, \tau\right)\right),
$$

and the first-order correction is $H_{1}=\partial_{\boldsymbol{r}_{c}} H_{c} \cdot\left(\boldsymbol{r}-\boldsymbol{r}_{c}\right)$. Here we have

$$
\dot{\phi}_{v}=\omega_{v}+\boldsymbol{Q}_{v}^{\prime} \cdot \dot{\boldsymbol{r}}_{c}
$$

which depends on the velocity of the wave packet. Because $H_{c}$ has the same translation symmetry as $H_{0}$, its eigenstates are still the Bloch states of $H_{0}$ as

$$
\left|\psi_{n \boldsymbol{k}}(\tilde{\boldsymbol{r}})\right\rangle=e^{i k_{\mu} \tilde{r}^{\mu}}\left|u_{n \boldsymbol{k}}(\tilde{\boldsymbol{r}})\right\rangle,
$$

but with a shifted position $\tilde{r}^{\mu}=r^{\mu}+\phi_{v} / Q_{v \mu}$ due to $\phi_{v}\left(\boldsymbol{r}_{c}, \tau\right)$.

The wave packet to the leading order can be constructed from the Bloch states as

$$
\left|W_{n}\right\rangle=\int d \boldsymbol{k} w_{n}(\boldsymbol{k}, \tau)\left|\psi_{n \boldsymbol{k}}(\tilde{\boldsymbol{r}})\right\rangle,
$$

where the electron dynamics is confined in a single band whose dispersion is $E_{n}(\boldsymbol{k})$, with $n$ being the band index. The interband coupling is encoded in the higher-order corrections and will be studied in the next section. In the following, we denote $\boldsymbol{r}_{n}$ and $\boldsymbol{k}^{n}$ as the center of mass of the wave packet $\left|W_{n}\right\rangle$ in the phase space. To be self-consistent, we have

$$
k_{\mu}^{n}=\left\langle W_{n}\left|k_{\mu}\right| W_{n}\right\rangle=\int d \boldsymbol{k}\left|w_{n}(\boldsymbol{k}, \tau)\right|^{2} k_{\mu},
$$

which indicates that $w_{n}(\boldsymbol{k}, \tau)$ is highly localized at $\boldsymbol{k}^{n}$, and

$$
\begin{aligned}
r_{n}^{\mu} & =\left\langle W_{n}\left|r^{\mu}\right| W_{n}\right\rangle \\
& =\int d \boldsymbol{k}^{\prime} \int d \boldsymbol{k} w_{n}^{*}\left(\boldsymbol{k}^{\prime}, \tau\right) w_{n}(\boldsymbol{k}, \tau)\left\langle\psi_{n \boldsymbol{k}^{\prime}}(\tilde{\boldsymbol{r}})\left|\left(-i \partial_{k_{\mu}} e^{i k_{\gamma} r^{\gamma}}\right) e^{i k_{\gamma} \phi_{v} / Q_{v \gamma}}\right| u_{n k}(\tilde{\boldsymbol{r}})\right\rangle \\
& =-i \int d \boldsymbol{k}^{\prime} \int d \boldsymbol{k} w_{n}^{*}\left(\boldsymbol{k}^{\prime}, \tau\right) w_{n}(\boldsymbol{k}, \tau)\left\langle\psi_{n \boldsymbol{k}^{\prime}}\left|\partial_{k_{\mu}}\right| \psi_{n \boldsymbol{k}}\right\rangle-\phi_{\nu} / Q_{v \mu}+i \int d \boldsymbol{k}^{\prime} \int d \boldsymbol{k} w_{n}^{*}\left(\boldsymbol{k}^{\prime}, \tau\right) w_{n}(\boldsymbol{k}, \tau)\left\langle\psi_{n \boldsymbol{k}^{\prime}}\left|e^{i k_{\gamma} \tilde{r}^{\gamma}}\right| \partial_{k_{\mu}} u_{n k}\right\rangle \\
& =-\partial_{k_{\mu}^{n}} \arg \left[w\left(\boldsymbol{k}^{n}, \tau\right)\right]-\phi_{\nu} / Q_{v \mu}+i\left\langle u_{n \boldsymbol{k}^{n}} \mid \partial_{k_{\mu}^{n}} u_{n \boldsymbol{k}^{n}}\right\rangle,
\end{aligned}
$$


where we have used the integration by parts and the fact that

$$
\begin{aligned}
\left\langle\psi_{n \boldsymbol{k}^{\prime}}(\tilde{\boldsymbol{r}})\left|e^{i k_{\gamma} \tilde{r}^{\gamma}}\right| \partial_{k_{\mu}} u_{n \boldsymbol{k}}(\tilde{\boldsymbol{r}})\right\rangle & =\frac{1}{N} \sum_{\boldsymbol{R}} e^{i\left(\boldsymbol{k}-\boldsymbol{k}^{\prime}\right) \cdot \boldsymbol{R}} \frac{1}{s_{0}} \int_{\mathrm{UC}} d \xi u_{n \boldsymbol{k}^{\prime}}(\boldsymbol{\xi}) e^{i\left(\boldsymbol{k}-\boldsymbol{k}^{\prime}\right) \cdot \boldsymbol{\xi}} \partial_{k_{\mu}} u_{n \boldsymbol{k}}(\boldsymbol{\xi}) \\
& =\delta\left(\boldsymbol{k}-\boldsymbol{k}^{\prime}\right)\left\langle u_{n \boldsymbol{k}^{\prime}}(\boldsymbol{\xi})\left|e^{i\left(\boldsymbol{k}-\boldsymbol{k}^{\prime}\right) \cdot \boldsymbol{\xi}}\right| \partial_{k_{\mu}} u_{n \boldsymbol{k}}(\boldsymbol{\xi})\right\rangle .
\end{aligned}
$$

Here, $N$ is the number of unit cells (UCs), $s_{0}$ is the area of a UC, and we parametrize $\tilde{\boldsymbol{r}}=\boldsymbol{R}+\boldsymbol{\xi}$, where $\boldsymbol{R}$ is the lattice vector and $\boldsymbol{\xi}$ is within the UC centered at the origin. Therefore, $\int d \tilde{\boldsymbol{r}}=\sum_{\boldsymbol{R}} \int_{\mathrm{UC}} d \boldsymbol{\xi}$ since $u_{n \boldsymbol{k}}(\boldsymbol{R}+\boldsymbol{\xi})=u_{n \boldsymbol{k}}(\boldsymbol{\xi})$.

The equation of motion can be obtained from the Lagrangian $L=\left\langle W_{n}\left|i \hbar \partial_{\tau}-H\right| W_{n}\right\rangle$. For the first term, we have

$$
\begin{aligned}
\left\langle W_{n}\left|i \hbar \partial_{\tau}\right| W_{n}\right\rangle & =\int d \boldsymbol{k}^{\prime} \int d \boldsymbol{k} w_{n}^{*}\left(\boldsymbol{k}^{\prime}, \tau\right)\left\langle\psi_{n \boldsymbol{k}^{\prime}}(\tilde{\boldsymbol{r}})\left|i \hbar \partial_{\tau} w_{n}(\boldsymbol{k}, \tau) e^{i k_{\mu} \tilde{r}^{\mu}}\right| u_{n \boldsymbol{k}}(\tilde{\boldsymbol{r}})\right\rangle \\
& =i \hbar \int d \boldsymbol{k} w_{n}^{*}(\boldsymbol{k}, \tau) \partial_{\tau} w_{n}(\boldsymbol{k}, \tau)-\hbar \int d \boldsymbol{k}\left|w_{n}(\boldsymbol{k}, \tau)\right|^{2} k_{\mu} \dot{\phi}_{\nu} / Q_{\nu \mu}+i \hbar \int d \boldsymbol{k}\left|w_{n}(\boldsymbol{k}, \tau)\right|^{2}\left\langle u_{n \boldsymbol{k}}(\tilde{\boldsymbol{r}}) \mid \partial_{\tau} u_{n \boldsymbol{k}}(\tilde{\boldsymbol{r}})\right\rangle \\
& =-\hbar \partial_{\tau} \arg \left[w_{n}\left(\boldsymbol{k}^{n}, \tau\right)\right]-\hbar k_{\mu}^{n} \dot{\phi}_{\nu} / Q_{\nu \mu}+i \hbar \dot{\phi}_{\nu}\left\langle u_{n \boldsymbol{k}^{n}} \mid \partial_{\phi_{\nu}} u_{n \boldsymbol{k}^{n}}\right\rangle \\
& =\hbar \dot{k}_{\mu}^{n} \partial_{\boldsymbol{k}_{\mu}^{n}} \arg \left[w_{n}\left(\boldsymbol{k}^{n}, \tau\right)\right]-\hbar k_{\mu}^{n} \dot{\phi}_{\nu} / Q_{\nu \mu}+i \hbar \dot{\phi}_{\nu}\left\langle u_{n \boldsymbol{k}^{n}} \mid \partial_{\phi_{\nu}} u_{n \boldsymbol{k}^{n}}\right\rangle
\end{aligned}
$$

where we have used the fact that $-\hbar \partial_{\tau} \arg \left[w_{n}\left(\boldsymbol{k}^{n}, \tau\right)\right]=$ $-\hbar \frac{d}{d t} \arg \left[w_{n}\left(\boldsymbol{k}^{n}, \tau\right)\right]+\hbar \dot{k}_{\mu}^{n} \partial_{k_{\mu}^{n}} \arg \left[w_{n}\left(\boldsymbol{k}^{n}, \tau\right)\right]$ and the total time derivative is dropped because it just contributes a constant to the action and does not affect the equation of motion. To the leading order, the second term of the Lagrangian is

$$
\left\langle W_{n}|H| W_{n}\right\rangle \approx\left\langle W_{n}\left|H_{c}\right| W_{n}\right\rangle=E_{n}\left(\boldsymbol{k}^{n}\right) .
$$

Now we combine Eqs. (A8) and (A10) together, which yields the final expression of the Lagrangian,

$$
\begin{aligned}
& L\left(\boldsymbol{r}_{n}, \dot{\boldsymbol{r}}_{n}, \boldsymbol{k}^{n}, \dot{\boldsymbol{k}}^{n}\right) \\
& \quad=\hbar \dot{k}_{\mu}^{n}\left(-r_{n}^{\mu}-\phi_{\nu} / Q_{\nu \mu}+i\left\langle u_{n \boldsymbol{k}^{n}} \mid \partial_{k_{\mu}^{n}} u_{n \boldsymbol{k}^{n}}\right\rangle\right) \\
& \quad-\hbar k_{\mu}^{n} \dot{\phi}_{\nu} / Q_{\nu \mu}+i \hbar \dot{\phi}_{\nu}\left\langle u_{n \boldsymbol{k}^{n}} \mid \partial_{\phi_{\nu}} u_{n \boldsymbol{k}^{n}}\right\rangle-E_{n}\left(\boldsymbol{k}^{n}\right) .
\end{aligned}
$$

According to the Euler-Lagrange equation, the leading-order semiclassical equation of the motion is

$$
\begin{aligned}
& \dot{r}_{n}^{\mu}=\frac{\partial E_{n}\left(\boldsymbol{k}^{n}\right)}{\hbar \partial k_{\mu}^{n}}-\dot{k}_{\gamma}^{n} \Omega_{n, k k}^{\mu \gamma}-\dot{\phi}_{\nu} \Omega_{n, k \phi}^{\mu \nu}, \\
& \dot{k}_{\mu}^{n}=0,
\end{aligned}
$$

where the Berry curvatures,

$$
\begin{aligned}
& \Omega_{n}^{\mu \gamma}=i\left\langle\partial_{k_{\mu}^{n}} u_{n \boldsymbol{k}^{n}} \mid \partial_{k_{\gamma}^{n}} u_{n} \boldsymbol{k}^{n}\right\rangle-i\left\langle\partial_{k_{\gamma}^{n}} u_{n} \boldsymbol{k}^{n} \mid \partial_{k_{\mu}^{n}} u_{n \boldsymbol{k}^{n}}\right\rangle, \\
& \Omega_{n}^{\mu \nu}=i\left\langle\partial_{k_{\mu}^{n}} u_{n \boldsymbol{k}^{n}} \mid \partial_{\phi_{\nu}} u_{n \boldsymbol{k}^{n}}\right\rangle-i\left\langle\partial_{\phi_{\nu}} u_{n \boldsymbol{k}^{n}} \mid \partial_{k_{\mu}^{n}} u_{n \boldsymbol{k}^{n}}\right\rangle,
\end{aligned}
$$

are, respectively, defined in the real momentum space and the hybrid momentum space. The dynamics of $\phi_{v}$ follows Eq. (A4), which is part of the equation of motion. The anomalous velocity $-\dot{\phi}_{\nu} \Omega_{n}^{\mu \nu}$ is due to the translational motion of the periodically modulated potential.

To study the higher-order corrections to the equation of motion, we consider the wave packet with the first-order corrections. According to the perturbation theory, the eigenstate of $H_{c}+H_{1}$ up to the first order is

$$
\begin{aligned}
\left|\psi_{n \boldsymbol{k}}(\tilde{\boldsymbol{r}})\right\rangle & =e^{i k_{\mu} \tilde{r}^{\mu}}\left[\left|u_{n \boldsymbol{k}}(\tilde{\boldsymbol{r}})\right\rangle+\sum_{m \neq n} c_{m}(\boldsymbol{k})\left|u_{m \boldsymbol{k}}(\tilde{\boldsymbol{r}})\right\rangle\right] \\
& =e^{i k_{\mu} \tilde{r}^{\mu}}\left[\left|u_{n \boldsymbol{k}}(\tilde{\boldsymbol{r}})\right\rangle+\left|u_{n \boldsymbol{k}}^{(1)}(\tilde{\boldsymbol{r}})\right\rangle\right],
\end{aligned}
$$

where $\left|u_{n \boldsymbol{k}}^{(1)}(\tilde{\boldsymbol{r}})\right\rangle$ is the first-order correction to the eigenstate and

$$
c_{m}(\boldsymbol{k})=\frac{\left\langle u_{m \boldsymbol{k}}(\tilde{\boldsymbol{r}})\left|H_{1}\right| u_{n \boldsymbol{k}}(\tilde{\boldsymbol{r}})\right\rangle}{E_{n}(\boldsymbol{k})-E_{m}(\boldsymbol{k})} .
$$

Thus the wave packet should be constructed from the corrected eigenstates as

$$
\begin{aligned}
\left|W_{n}\right\rangle & =\left|W_{n}^{(0)}\right\rangle+\left|W_{n}^{(1)}\right\rangle \\
& =\int d \boldsymbol{k} w_{n}(\boldsymbol{k}, \tau) e^{i k_{\mu} \tilde{r}^{\mu}}\left[\left|u_{n \boldsymbol{k}}(\tilde{\boldsymbol{r}})\right\rangle+\left|u_{n \boldsymbol{k}}^{(1)}(\tilde{\boldsymbol{r}})\right|\right] .
\end{aligned}
$$

The self-consistent conditions of the wave packet are still $k_{\mu}^{n}=\left\langle W_{n}\left|k_{\mu}\right| W_{n}\right\rangle$ and

$$
r_{n}^{\mu}=\left\langle W_{n}^{(0)}\left|r^{\mu}\right| W_{n}^{(0)}\right\rangle+\left\langle W_{n}^{(0)}\left|r^{\mu}\right| W_{n}^{(1)}\right\rangle+\left\langle W_{n}^{(1)}\left|r^{\mu}\right| W_{n}^{(0)}\right\rangle,
$$

up to the first order. Here the first term is the same as Eq. (A8) and the second term is

$$
\left\langle W_{n}^{(0)}\left|r^{\mu}\right| W_{n}^{(1)}\right\rangle=i\left\langle u_{n k^{n}} \mid \partial_{k_{\mu}^{n}} u_{n k^{n}}^{(1)}\right\rangle,
$$

where we have used the fact that $\left\langle u_{n k} \mid u_{n k}^{(1)}\right\rangle=0$. Therefore, the center of mass is

$$
\begin{aligned}
r_{n}^{\mu}= & -\partial_{k_{\mu}^{n}} \arg \left[w_{n}\left(\boldsymbol{k}^{n}, \tau\right)\right]-\phi_{\nu} / Q_{\nu \mu}+i\left\langle u_{n \boldsymbol{k}^{n}} \mid \partial_{k_{\mu}^{n}} u_{n \boldsymbol{k}^{n}}\right\rangle \\
& +i\left\langle u_{n \boldsymbol{k}^{n}} \mid \partial_{k_{\mu}^{n}} u_{n \boldsymbol{k}^{n}}^{(1)}\right\rangle+i\left\langle u_{n \boldsymbol{k}^{n}}^{(1)} \mid \partial_{k_{\mu}^{n}} u_{n \boldsymbol{k}^{n}}\right\rangle,
\end{aligned}
$$

and the first-order corrections come from the interband coupling.

For the Lagrangian $L=\left\langle W_{n}\left|i \hbar \partial_{\tau}-H\right| W_{n}\right\rangle$, the first term now becomes

$$
\begin{aligned}
\left\langle W_{n}\left|i \hbar \partial_{\tau}\right| W_{n}\right\rangle= & \left\langle W_{n}^{(0)}\left|i \hbar \partial_{\tau}\right| W_{n}^{(0)}\right\rangle+\left\langle W_{n}^{(0)}\left|i \hbar \partial_{\tau}\right| W_{n}^{(1)}\right\rangle \\
& +\left\langle W_{n}^{(1)}\left|i \hbar \partial_{\tau}\right| W_{n}^{(0)}\right\rangle,
\end{aligned}
$$

where the leading-order term $\left\langle W_{n}^{(0)}\left|i \hbar \partial_{\tau}\right| W_{n}^{(0)}\right\rangle$ is the same as Eq. (A10) and the first-order correction is

$$
\left\langle W_{n}^{(0)}\left|i \hbar \partial_{\tau}\right| W_{n}^{(1)}\right\rangle=i \hbar \dot{\phi}_{\nu}\left\langle u_{n \boldsymbol{k}_{n}} \mid \partial_{\phi_{\nu}} u_{n \boldsymbol{k}_{n}}^{(1)}\right\rangle .
$$


The dispersion relation is corrected to the first order as

$$
\left\langle W_{n}|H| W_{n}\right\rangle \approx\left\langle W_{n}\left|H_{c}+H_{1}\right| W_{n}\right\rangle=\tilde{E}_{n}\left(\boldsymbol{k}^{n}\right)
$$

Now the Lagrangian becomes

$$
\begin{aligned}
L\left(\boldsymbol{r}_{n}, \dot{\boldsymbol{r}}_{n}, \boldsymbol{k}^{n}, \dot{\boldsymbol{k}}^{n}\right)= & \hbar \dot{k}_{\mu}^{n} \partial_{k_{\mu}^{n}} \arg \left[w_{n}\left(\boldsymbol{k}^{n}, \tau\right)\right]-\hbar k_{\mu}^{n} \dot{\phi}_{\nu} / Q_{\nu \mu}+i \hbar \dot{\phi}_{\nu}\left\langle u_{n \boldsymbol{k}^{n}} \mid \partial_{\phi_{\nu}} u_{\boldsymbol{k}^{n}}\right\rangle+i \hbar \dot{\phi}_{\nu}\left\langle u_{n \boldsymbol{k}_{n}} \mid \partial_{\phi_{v}} u_{n \boldsymbol{k}_{n}}^{(1)}\right\rangle+i \hbar \dot{\phi}_{\nu}\left\langle u_{n \boldsymbol{k}_{n}}^{(1)} \mid \partial_{\phi_{\nu}} u_{n \boldsymbol{k}_{n}}\right\rangle \\
= & \hbar \dot{k}_{\mu}^{n}\left(-r_{n}^{\mu}-\phi_{\nu} / Q_{\nu \mu}+i\left\langle u_{n \boldsymbol{k}^{n}} \mid \partial_{k_{\mu}^{n}} u_{n \boldsymbol{k}^{n}}\right\rangle+i\left\langle u_{n \boldsymbol{k}^{n}} \mid \partial_{k_{\mu}^{n}} u_{n \boldsymbol{k}^{n}}^{(1)}\right\rangle+i\left\langle u_{n \boldsymbol{k}^{n}}^{(1)} \mid \partial_{k_{\mu}^{n}} u_{n \boldsymbol{k}^{n}}\right\rangle\right)-\hbar k_{\mu}^{n} \dot{\phi}_{\nu} / Q_{\nu \mu} \\
& +i \hbar \dot{\phi}_{\nu}\left\langle u_{n \boldsymbol{k}^{n}} \mid \partial_{\phi_{\nu}} u_{\boldsymbol{k}^{n}}\right\rangle+i \hbar \dot{\phi}_{\nu}\left\langle u_{n \boldsymbol{k}_{n}} \mid \partial_{\phi_{\nu}} u_{n \boldsymbol{k}_{n}}^{(1)}\right\rangle+i \hbar \dot{\phi}_{\nu}\left\langle u_{n \boldsymbol{k}_{n}}^{(1)} \mid \partial_{\phi_{\nu}} u_{n \boldsymbol{k}_{n}}\right\rangle
\end{aligned}
$$

according to Eq. (A21). The Euler-Lagrange equation yields the semiclassical equation of motion with the higher-order corrections as

$$
\begin{aligned}
& \dot{r}_{n}^{\mu}=\frac{\partial \tilde{E}_{n}\left(\boldsymbol{k}^{n}\right)}{\hbar \partial k_{\mu}^{n}}-\dot{k}_{\gamma}^{n} \tilde{\Omega}_{n, k k}^{\mu \gamma}-\dot{\phi}_{\nu} \tilde{\Omega}_{n, k \phi}^{\mu \nu}, \\
& \dot{k}_{\mu}^{n}=0,
\end{aligned}
$$

where the Berry curvatures with the higher-order corrections are

$$
\begin{gathered}
\tilde{\Omega}_{n}^{\mu \gamma}=\Omega_{n}^{\mu \gamma}+i\left(\partial_{k_{\mu}^{n}}\left\langle u_{n \boldsymbol{k}^{n}} \mid \partial_{k_{\gamma}^{n}} u_{n \boldsymbol{k}^{n}}^{(1)}\right\rangle-\partial_{k_{\gamma}^{n}}\left\langle u_{n \boldsymbol{k}^{n}} \mid \partial_{k_{\mu}^{n}} u_{n \boldsymbol{k}^{n}}^{(1)}\right\rangle\right)+i\left(\partial_{k_{\mu}^{n}}\left\langle u_{n \boldsymbol{k}^{n}}^{(1)} \mid \partial_{k_{\gamma}^{n}} u_{n \boldsymbol{k}^{n}}\right\rangle-\partial_{k_{\gamma}^{n}}\left\langle u_{n \boldsymbol{k}^{n}}^{(1)} \mid \partial_{k_{\mu}^{n}} u_{n \boldsymbol{k}^{n}}\right\rangle\right), \\
\tilde{\Omega}_{n}^{\mu \nu}=\Omega_{n}^{\mu \nu}+i\left(\partial_{k_{\mu}^{n}}\left\langle u_{n} \mid \partial_{\phi_{\nu}} u_{n \boldsymbol{k}^{n}}^{(1)}\right\rangle-\partial_{\phi_{\nu}}\left\langle u_{n \boldsymbol{k}^{n}} \mid \partial_{k_{\mu}^{n}} u_{n \boldsymbol{k}^{n}}^{(1)}\right\rangle\right)+i\left(\partial_{k_{\mu}^{n}}\left\langle u_{n \boldsymbol{k}^{n}}^{(1)} \mid \partial_{\phi_{\nu}} u_{n \boldsymbol{k}^{n}}\right\rangle-\partial_{\phi_{\nu}}\left\langle u_{n \boldsymbol{k}^{n}}^{(1)} \mid \partial_{k_{\mu}^{n}} u_{n \boldsymbol{k}^{n}}\right\rangle\right) .
\end{gathered}
$$

Apparently, the equation of motion with the higher-order corrections is still in the same form as the leading-order equation of motion derived above. The modified dispersion relation and Berry curvature with first-order corrections are induced by the interband coupling. Because the higher-order correction does not affect the response function, as proved in the next section, it is not included in Eq. (3). In the main text and hereafter, the band index $n$ of crystal momentum is dropped for simplicity.

\section{APPENDIX B: NONLINEAR RESPONSE FUNCTION}

The current density due to the motion and deformation of periodic modulation potential (induced by spin textures) is

$$
j^{\mu}=e \sum_{n} \int d^{2} k \dot{r}_{n}^{\mu} f\left(E_{n}\right) D_{n}(\boldsymbol{r}, \tilde{\boldsymbol{k}}),
$$

where $D_{n}(\boldsymbol{r}, \tilde{\boldsymbol{k}})$ is the phase-space density of states (PSDOS). In the absence of deformation, the phase space is homogeneous and the PSDOS is a constant as $D_{n}=1 /(2 \pi)^{d}$, where $d$ is the dimension of the system. However, due to the deformation, the phase space is inhomogeneous and we need to consider the modification of the PSDOS. The basic idea is to study the time evolution of phase-space volume element $\Delta V=\Delta \boldsymbol{r} \Delta \tilde{\boldsymbol{k}}$ that follows $(1 / \Delta V) d \Delta V / d t=\nabla_{\boldsymbol{r}} \cdot \dot{\boldsymbol{r}}+\nabla_{\tilde{\boldsymbol{k}}} \cdot \dot{\tilde{\boldsymbol{k}}}$ [51]. The equation can be solved [52], which yields the modified PSDOS,

$$
D_{n}(\boldsymbol{r}, \tilde{\boldsymbol{k}})=\frac{1}{\Delta V}=\frac{1}{(2 \pi)^{2}}\left(1+\frac{1}{2} Q_{\mu \nu}^{\prime} \tilde{\Omega}_{n}^{v \mu}+\frac{1}{64} \varepsilon^{\mu \nu \gamma \delta} Q_{\mu \nu}^{\prime} Q_{\gamma \delta}^{\prime} \varepsilon_{\eta \rho \alpha \beta} \tilde{\Omega}_{n}^{\eta \rho} \tilde{\Omega}_{n}^{\alpha \beta}\right),
$$

where $\varepsilon^{\mu \nu \gamma \delta}$ and $\varepsilon_{\eta \rho \alpha \beta}$ are the Levi-Civita tensor with $\varepsilon^{x y 12}=\varepsilon_{x y 12}=1$. Plugging Eq. (A4) into Eq. (A26) and retaining the terms up to the second order in $\omega_{\nu}$ and $Q_{\nu \mu}^{\prime}$ yields

$$
\dot{r}_{n}^{\mu}=\frac{\partial \tilde{E}_{n}}{\hbar \partial k_{\mu}}-\omega_{\nu} \tilde{\Omega}_{n}^{\mu \nu}-\left(\frac{\partial \tilde{E}_{n}}{\hbar \partial k_{\gamma}}-\omega_{\delta} \tilde{\Omega}_{n}^{\gamma \delta}-\frac{\partial \tilde{E}_{n}}{\hbar \partial k_{\eta}} Q_{\delta \eta}^{\prime} \tilde{\Omega}_{n}^{\gamma \delta}\right) Q_{\nu \gamma}^{\prime} \tilde{\Omega}_{n}^{\mu \nu},
$$

which is in the same form of Eq. (8), but with modified dispersion relation and Berry curvature due to the first-order corrections. In the following, we will show that our final results are not modified by the first-order corrections, which therefore can be dropped. Now we substitute Eqs. (B2) and (B3) into Eq. (B1) and retain the terms up to the second order in $\omega_{\nu}$ and $Q_{v \mu}^{\prime}$. We then have

$$
\begin{aligned}
j^{\mu}= & e \sum_{n} \int \frac{d^{2} k}{(2 \pi)^{2}} f\left(\tilde{E}_{n}-E_{F}\right)\left[\left(\frac{\partial \tilde{E}_{n}}{\hbar \partial k_{\mu}}-\omega_{\nu} \tilde{\Omega}_{n}^{\mu \nu}\right)\right. \\
& +\left(\omega_{\delta} \Omega_{n}^{\gamma \delta} Q_{\nu \gamma}^{\prime} \Omega_{n}^{\mu \nu}-\frac{1}{2} \omega_{\nu} \Omega_{n}^{\mu \nu} Q_{\gamma \delta}^{\prime} \Omega_{n}^{\delta \gamma}\right)-\left(\frac{\partial \tilde{E}_{n}}{\hbar \partial k_{\gamma}} Q_{\nu \gamma}^{\prime} \tilde{\Omega}_{n}^{\mu \nu}-\frac{1}{2} \frac{\partial \tilde{E}_{n}}{\hbar \partial k_{\mu}} Q_{\gamma \delta}^{\prime} \tilde{\Omega}_{n}^{\delta \gamma}\right) \\
& \left.+\left(\frac{\partial E_{n}}{\hbar \partial k_{\eta}} Q_{\delta \eta}^{\prime} \Omega_{n}^{\gamma \delta} Q_{\nu \gamma}^{\prime} \Omega_{n}^{\mu \nu}-\frac{1}{2} \frac{\partial E_{n}}{\hbar \partial k_{\gamma}} Q_{\nu \gamma}^{\prime} \Omega_{n}^{\mu \nu} Q_{\eta \delta}^{\prime} \Omega_{n}^{\delta \eta}+\frac{1}{64} \frac{\partial E_{n}}{\hbar \partial k_{\mu}} \varepsilon^{\lambda \nu \gamma \delta} Q_{\lambda \nu}^{\prime} Q_{\gamma \delta}^{\prime} \varepsilon_{\eta \rho \alpha \beta} \Omega_{n}^{\eta \rho} \Omega_{n}^{\alpha \beta}\right)\right] .
\end{aligned}
$$


Here we collect different terms with similar orders and forms into four groups enclosed by brackets. The integral of the first group just gives Eq. (4) where higher-order corrections are dropped. Since we focus on the Fermi energy in the bulk energy gap that remains open upon weak perturbation, the modified group velocity $\partial_{k_{\mu}} \tilde{E}_{n} / \hbar$ and the first-order correction to the Berry curvature $\Omega_{n}^{(1) \mu \nu}$ vanish under the integration over the hybrid Brillouin zone [53]. Therefore, the transported charge described by Eq. (5) is unaffected by the higher-order corrections. Notably, the second group in Eq. (B4) is unaffected by the higher-order corrections. Because $\Omega_{n}^{\mu \nu}=-\Omega_{n}^{v \mu}$, $Q_{\mu \nu}^{\prime}=-Q_{\nu \mu}^{\prime}$, and the indices can be freely replaced, we have

$$
\begin{aligned}
\omega_{\delta} \Omega_{n}^{\gamma \delta} Q_{\nu \gamma}^{\prime} \Omega_{n}^{\mu \nu}-\frac{1}{2} \omega_{\nu} \Omega_{n}^{\mu \nu} Q_{\gamma \delta}^{\prime} \Omega_{n}^{\delta \gamma} \\
=\frac{\omega_{\nu} Q_{\gamma \delta}^{\prime}}{2}\left(\Omega_{n}^{\mu \nu} \Omega_{n}^{\gamma \delta}+\Omega_{n}^{\mu \gamma} \Omega_{n}^{\delta \nu}+\Omega_{n}^{\mu \delta} \Omega_{n}^{\nu \gamma}\right),
\end{aligned}
$$

for the second group. Here we use the transformation $\omega_{\delta} \Omega_{n}^{\gamma \delta} Q_{\nu \gamma}^{\prime} \Omega_{n}^{\mu \nu} \rightarrow \omega_{\nu} \Omega_{n}^{\nu \gamma} Q_{\gamma \delta}^{\prime} \Omega_{n}^{\mu \delta} \quad$ under $\delta \leftrightarrow v$ and $\omega_{\delta} \Omega_{n}^{\gamma \delta} Q_{v \gamma}^{\prime} \Omega_{n}^{\mu \nu} \rightarrow \omega_{\nu} \Omega_{n}^{\delta v} Q_{\gamma \delta}^{\prime} \Omega_{n}^{\mu \gamma}$ under $\gamma \leftrightarrow \nu$ and then $\nu \leftrightarrow$ $\delta$. Therefore, the integral of the first two groups yields the nonlinear response function given by Eq. (9).

Next, we prove that the integral of the last two groups is zero. For the third group, we use the same trick as above and we obtain

$$
\begin{aligned}
& \frac{\partial \tilde{E}_{n}}{\hbar \partial k_{\gamma}} Q_{\nu \gamma}^{\prime} \tilde{\Omega}_{n}^{\mu \nu}-\frac{1}{2} \frac{\partial \tilde{E}_{n}}{\hbar \partial k_{\mu}} Q_{\gamma \delta}^{\prime} \tilde{\Omega}_{n}^{\delta \gamma} \\
& \quad=\frac{Q_{\gamma \delta}^{\prime}}{2 \hbar}\left(\frac{\partial \tilde{E}_{n}}{\partial k_{\mu}} \tilde{\Omega}_{n}^{\gamma \delta}+\frac{\partial \tilde{E}_{n}}{\partial k_{\gamma}} \tilde{\Omega}_{n}^{\delta \mu}+\frac{\partial \tilde{E}_{n}}{\partial k_{\delta}} \tilde{\Omega}_{n}^{\mu \gamma}\right) .
\end{aligned}
$$

For a fully occupied band at zero temperature, the integral of Eq. (B6) yields

$$
\frac{Q_{\gamma \delta}^{\prime}}{2 \hbar} \int d^{2} k \tilde{E}_{n}\left(\frac{\partial \tilde{\Omega}_{n}^{\gamma \delta}}{\partial k_{\mu}}+\frac{\partial \tilde{\Omega}_{n}^{\delta \mu}}{\partial k_{\gamma}}+\frac{\partial \tilde{\Omega}_{n}^{\mu \gamma}}{\partial k_{\delta}}\right),
$$

where we have used the integration by parts and the fact that both $\tilde{E}_{n}$ and $\tilde{\Omega}_{n}^{\mu \nu}$ are periodic over the Brillouin zone. The three terms in the bracket are equal to zero according to the Bianchi identity [54]. Therefore, the third group in Eq. (B4) does not contribute to the current density. The last group vanishes due to the antisymmetric property of $\Omega_{n}^{\mu \nu}$ and $Q_{\mu \nu}^{\prime}$. To show that the three terms are indeed canceled out, we take $j^{y}$ as an example and we collect all the terms associated with $\frac{\partial E_{n}}{\hbar \partial k_{y}} Q_{1 y}^{\prime} Q_{2 x}^{\prime}$. Here the first two terms give

$$
\frac{\partial E_{n}}{\hbar \partial k_{y}} Q_{1 y}^{\prime} Q_{2 x}^{\prime}\left(\Omega_{n}^{x 1} \Omega_{n}^{y 2}-\Omega_{n}^{y 1} \Omega_{n}^{x 2}\right),
$$

and the last term yields

$$
\frac{\partial E_{n}}{\hbar \partial k_{y}} Q_{1 y}^{\prime} Q_{2 x}^{\prime}\left(-\Omega_{n}^{x 1} \Omega_{n}^{y 2}+\Omega_{n}^{y 1} \Omega_{n}^{x 2}\right),
$$

where we have used the property $\Omega_{n}^{\mu \nu} Q_{\gamma \delta}^{\prime}=\Omega_{n}^{v \mu} Q_{\delta \gamma}^{\prime}$, $\Omega_{n}^{\mu \nu} \Omega_{n}^{\gamma \delta}=\Omega_{n}^{\nu \mu} \Omega_{n}^{\delta \gamma}$, and $Q_{\mu \nu}^{\prime} Q_{\gamma \delta}^{\prime}=Q_{\nu \mu}^{\prime} Q_{\delta \gamma}^{\prime}$. Obviously, Eqs. (B8) and (B9) have the opposite sign, and hence the last group has no contribution to the current density.

\section{APPENDIX C: MONTE CARLO SIMULATION}

We present details of the model and numerical simulations for the spin textures. Here we consider two models in order to describe three multiple- $Q$ states: one is the effective spin model derived from the Kondo lattice model for the double$Q$ and triple- $Q$ collinear spin textures and the other is the frustrated spin model for the triple- $Q$ skyrmion spin texture.

The effective spin model is given by $[55,56]$

$$
\begin{aligned}
\mathcal{H}= & 2 \sum_{v}\left[-\tilde{J}\left\{\alpha\left(S_{\boldsymbol{Q}_{v}}^{x} S_{-\boldsymbol{Q}_{v}}^{x}+S_{\boldsymbol{Q}_{v}}^{y} S_{-\boldsymbol{Q}_{v}}^{y}\right)+S_{\boldsymbol{Q}_{v}}^{z} S_{-\boldsymbol{Q}_{v}}^{z}\right\}\right. \\
& \left.+\tilde{K}\left\{\alpha\left(S_{\boldsymbol{Q}_{v}}^{x} S_{-\boldsymbol{Q}_{v}}^{x}+S_{\boldsymbol{Q}_{v}}^{y} S_{-\boldsymbol{Q}_{v}}^{y}\right)+S_{\boldsymbol{Q}_{v}}^{z} S_{-\boldsymbol{Q}_{v}}^{z}\right\}^{2}\right] \\
& -A \sum_{i}\left(S_{i}^{z}\right)^{2},
\end{aligned}
$$

where $\boldsymbol{S}_{\boldsymbol{q}}$ is the Fourier transform of $\boldsymbol{S}_{i}$ and $\boldsymbol{Q}_{v}[v=1,2$ $(1,2,3)$ for the square (triangular) lattice] are the equivalent wave vectors for the multiple peaks in the bare susceptibility of itinerant electrons. The bilinear interaction $\tilde{J}=1$ represents the effective Ruderman-Kittel-Kasuya-Yosida interaction, and the positive biquadratic interaction $K=N \tilde{K}$, with $N$ the number of sites, represents the four-spin interactions leading to the multiple- $Q$ instability. $\alpha$ is introduced as the anisotropy parameter for exchange coupling, where $\alpha=0$ represents the Ising-type coupling. The third term represents the easy-axis anisotropy $(A>0)$ for localized spins.

The simulations for the effective spin model are carried out with the classical Monte Carlo simulations at low temperatures. Our simulations are carried out with the standard Metropolis local updates. The results are obtained for systems with $N=96 \times 96$ sites under periodic boundary conditions. In each simulation, we perform simulated annealing to find the low-energy spin configuration by gradually reducing the temperature with the rate $T_{n+1}=a T_{n}$, where $T_{n}$ is the temperature in the $n$th step. We set the initial temperature $T_{0}=1.0-10.0$. We take $a=0.99995-0.99999$ and the final temperature is typically taken at $T=0.001-0.01$. We also start the simulations from the multiple- $Q$ spin configurations, such as the double- $Q$ and triple- $Q$ collinear spin textures. We determine the magnetic phase by comparing their energies of the obtained magnetic patterns in simulations. The double- $Q$ collinear spin texture in Fig. 1(a) is obtained at $T=0.01$, $K=0.5, \alpha=1.0, A=0.5$, and $|Q|=2 \pi / 6$ on the square lattice, while the triple- $Q$ collinear spin texture in Fig. 1(b) is obtained at $T=0.001, K=1.0, \alpha=0.0, A=0.0$, and $|\boldsymbol{Q}|=2 \pi / 6$ on the triangular lattice.

The frustrated spin model on the triangular lattice is given by [57]

$$
\mathcal{H}=\sum_{\langle i, j\rangle} J_{i j} \mathbf{S}_{i} \cdot \mathbf{S}_{j}-H \sum_{i} S_{i}^{z}-A \sum_{i}\left(S_{i}^{z}\right)^{2},
$$

where we consider the ferromagnetic nearest-neighbor coupling $J_{1}=-1$ and antiferromagnetic third-nearest-neighbor coupling $J_{3}=0.5$ in the first term, which gives the ordering vector $|\boldsymbol{Q}|=2 \pi / 5$. The second and third terms represent the Zeeman coupling to an external magnetic field and the easyaxis anisotropy, respectively.

The optimal magnetic phases in the frustrated spin model are obtained from the Monte Carlo simulations based on the 
Metropolis algorithm. The lattices have $N=100 \times 100$ spins and the periodic boundary conditions. In the simulations, the $10^{5}-10^{7}$ Monte Carlo sweeps measurements are performed after equilibration. The triple- $Q$ skyrmion spin texture in Fig. 1(c) is obtained at $T=0.01, H=0.27$, and $A=0.5$.

\section{APPENDIX D: TIME-DEPENDENT CURRENT}

In the lattice model, the current through a bond connecting lattice sites $i$ and $j$ is

$$
I_{i j}(\tau)=-i \frac{e}{\hbar} \sum_{E_{n} \leqslant E_{F}, \sigma}\left\langle\psi_{n}(\tau)\left|c_{i, \sigma}^{\dagger} t_{i j} c_{j, \sigma}-c_{j, \sigma}^{\dagger} t_{j i} c_{i, \sigma}\right| \psi_{n}(\tau)\right\rangle,
$$

where $\sigma=\uparrow$ or $\downarrow$ for spin degree of freedom and $\left|\psi_{n}(\tau)\right\rangle$ is the time evolution of the eigenstate $\left|\psi_{n}\right\rangle$ that is obtained by exact diagonalization of the Hamiltonian given by Eq. (1) at $\tau=0$. The time evolution of the eigenstates follows the timedependent Schrödinger equation $i \hbar \partial_{\tau}\left|\psi_{n}(\tau)\right\rangle=\mathcal{H}(\tau)\left|\psi_{n}(\tau)\right\rangle$, which is solved numerically by the Runge-Kutta method. The total current $I_{x}\left(I_{y}\right)$ is the sum of all the bond currents through a cross section that is perpendicular to the $x(y)$ direction.

\section{APPENDIX E: MAPPING TO A HIGH-DIMENSIONAL HOFSTADTER MODEL}

To compare with the numerical results for the spin textures from Monte Carlo simulation, we study the anomalous charge transport for the collinear spin texture ansatz described by

$$
\boldsymbol{S}_{i}=\left[0,0, \sum_{\nu} \cos \left(\boldsymbol{Q}_{\nu} \cdot \boldsymbol{r}_{i}+\phi_{\nu}\right)\right] \text {. }
$$

The spin texture ansatz is exactly periodic and hence wellquantized anomalous charge transport is expected. Interestingly, the Hamiltonian given by Eq. (1) with the spin texture can be mapped to a generalized high-dimensional Hofstadter model [58],

$$
\begin{aligned}
\mathcal{H}^{\prime}= & -t \sum_{x, y, k_{z}, k_{w}}\left(c_{x+1, y, k_{z}, k_{w}}^{\dagger} c_{x, y, k_{z}, k_{w}}\right. \\
& \left.+c_{x, y+1, k_{z}, k_{w}}^{\dagger} c_{x, y, k_{z}, k_{w}}+\text { H.c. }\right) \\
& -2 t \sum_{x, y, k_{z}, k_{w}}\left[\cos \left(B_{x z} x+B_{y z} y+k_{z}\right)\right. \\
& \left.+\cos \left(B_{x w} x+B_{y w} y+k_{w}\right)\right] c_{x, y, k_{z}, k_{w}}^{\dagger} c_{x, y, k_{z}, k_{w}},
\end{aligned}
$$

for electrons hopping on a hypercubic lattice in the 4D space spanned by $(x, y, z, w)$. Here we define the dimensionless magnetic field $B_{\mu \nu}$ such that the magnetic flux through a unit square in the $\mu \nu$ plane is $B_{\mu \nu} \Phi_{0}$, where $\Phi_{0}=h c / e$ is the quantum flux unit. Comparing Eq. (E2) with Eqs. (1) and (E1), one can find the correspondence that the phase $\phi_{v}$ plays the same role as the crystal momentum, $\phi_{1} \sim k_{z}$ and $\phi_{2} \sim k_{w}$. The $\boldsymbol{Q}_{v}$ vector corresponds to the magnetic field, $Q_{1 x} \sim B_{x z}, Q_{1 y} \sim B_{y z}, Q_{2 x} \sim B_{x w}$, and $Q_{2 y} \sim B_{y w}$, for the double $Q$ state. The motion of spin textures with $\dot{\phi}_{v}=\omega_{v}$ is analogous to the dynamical equation $\hbar \dot{k}_{\mu}=e E_{\mu}$, where $E_{\mu}$ is the component of the electric field. Hence, $\omega_{\nu}$ behaves like an electric field. According to the mapping, the
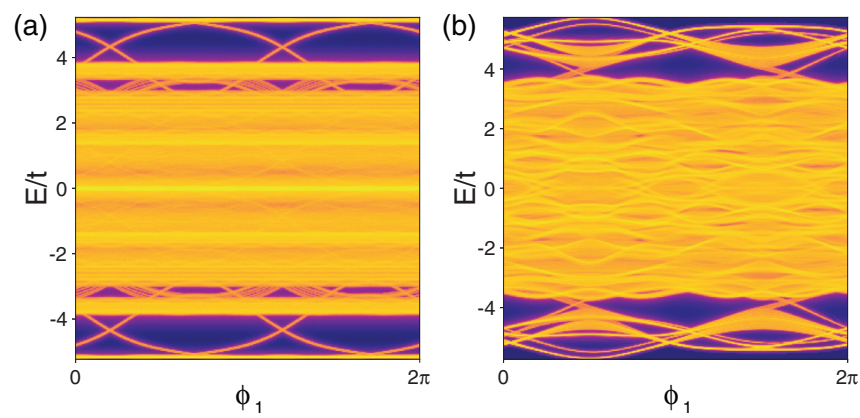

FIG. 4. Electronic spectral functions of the magnets with (a) the double- $Q$ collinear spin texture and (b) the triple- $Q$ collinear spin texture, depicted by the ansatz as a function of $\phi_{1}$. Here the purple (yellow) denotes the low (high) density of states.

high-dimensional nontrivial topology and quantum Hall effect can be understood intuitively [59]. To simulate the anomalous charge transport due to the motion of the spin texture ansatz, we consider a double- $Q$ collinear spin texture with $\boldsymbol{Q}_{1}=(2 \pi / a, 0)$ and $\boldsymbol{Q}_{2}=(0,2 \pi / a)$, and a triple- $Q$ collinear spin texture with $\boldsymbol{Q}_{1}=(-2 \pi / a, 2 \sqrt{3} \pi / a), \boldsymbol{Q}_{2}=$ $(-2 \pi / a,-2 \sqrt{3} \pi / a)$, and $\boldsymbol{Q}_{3}=(4 \pi / a, 0)$. We first study the electronic spectrum to identify the topological property of the system. For conduction electrons hopping on a square lattice (whose lattice constant is set to unity), we fix $a=5$ for both the double- $Q$ and triple- $Q$ spin textures; while for the triple- $Q$ spin texture, to make it commensurate with the lattice, we compress the lattice constant along the $y$ direction to $1 / \sqrt{3}$ such that each supercell also contains $5 \times 5$ lattice sites. The energy spectra as a function of $\phi_{1}$ for the double- $Q$ and triple$Q$ spin textures when $J=1.5 t$ and $B=0$ in Eq. (1) are shown in Figs. 4(a) and 4(b), respectively. Here we use the open boundary condition along the $x$ direction and the periodic boundary condition along the $y$ direction. Apparently, there are topological edge states in the bulk energy gaps indicating that the system is topologically nontrivial. To characterize the nontrivial topology, we calculate the Chern numbers of the system. Here we focus on the Fermi energy in the bottom bulk energy gaps in Fig. 4. In the 4D hybrid momentum space of the double- $Q$ spin texture, there are six first Chern numbers: $C_{1}^{x 1}=C_{1}^{y 2}=2$ and $C_{1}^{x y}=C_{1}^{x 2}=C_{1}^{y 1}=C_{1}^{12}=0$, and one second Chern number: $C_{2}^{x 1 y 2}=-2$. In the 5D hybrid momentum space of the triple- $Q$ spin texture, there are ten first Chern numbers: $C_{1}^{x 1}=C_{1}^{x 2}=-C_{1}^{y 1}=C_{1}^{y 2}=-2$ and $C_{1}^{x y}=C_{1}^{x 3}=$ $C_{1}^{y 3}=C_{1}^{12}=C_{1}^{13}=C_{1}^{23}=0$, and five second Chern numbers: $C_{2}^{x 1 y 2}=-2$ and $C_{2}^{x y 13}=C_{2}^{x y 23}=C_{2}^{x 123}=C_{2}^{y 123}=0$. The first Chern numbers on different 2D planes and second Chern numbers on different 4D hypersurfaces are independent of the other momenta because the bulk energy gaps are open in the entire hybrid momentum space.

We then study the anomalous charge transport induced by the translational motion of the spin textures, which is parameterized by $\phi_{\nu}=\omega_{\nu} \tau$. Here we consider the spin texture moving in the $y$ direction to be same as that in the main text. For the double- $Q$ spin texture, we have $\omega_{1}=0$ and $\omega_{2}=$ $2 \pi t / 100 \hbar$, while for the triple- $Q$ spin texture, we have $\omega_{1}=$ $-\omega_{2}=2 \pi t / 100 \hbar$ and $\omega_{3}=0$. Thus the period of motion of 


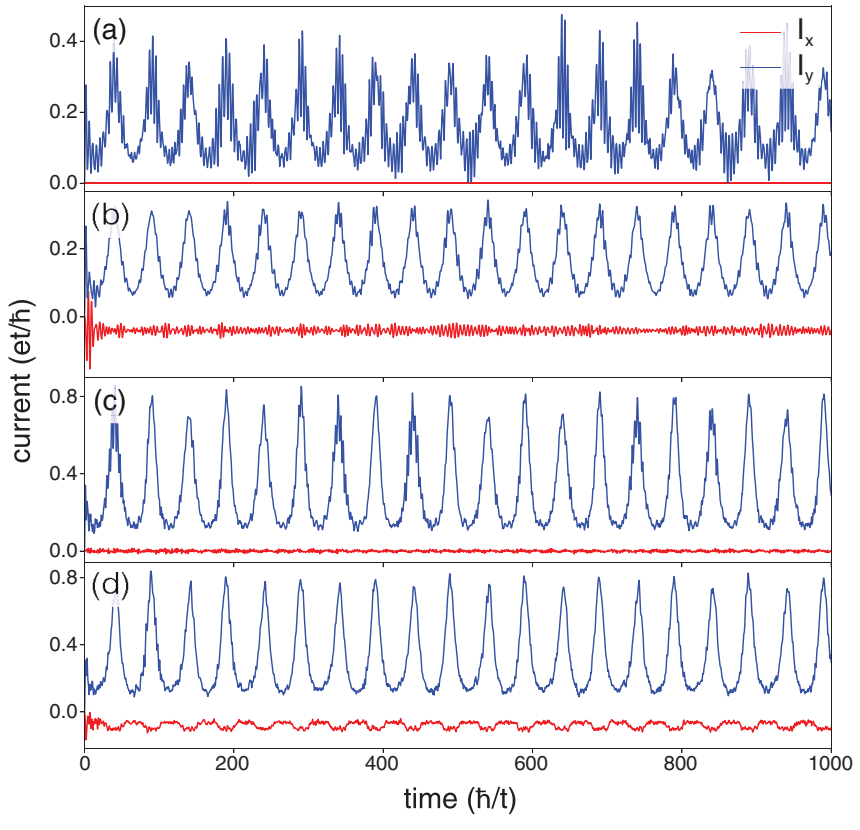

FIG. 5. Currents along the $x$ and $y$ directions for the magnets with multiple- $Q$ collinear spin textures depicted by the ansatz. (a),(b) Currents for the double- $Q$ spin texture without and with deformation, respectively. (c),(d) Currents for the triple- $Q$ spin texture without and with deformation, respectively.

both the double- $Q$ and triple- $Q$ spin textures is $\Delta \tau=100 \hbar / t$. We calculate the currents due to the motion of spin textures on a $40 \times 40$ lattice (containing $8 \times 8$ supercells). For the double- $Q$ spin texture, the currents over ten periods are shown in Fig. 5(a). By integrating the currents over time, the average transported charges in one period are quantized as $q^{x}=$ $0.00 e$ and $q^{y}=15.99 e$. According to Eq. (5), the transported charges are $q^{x}=e N^{x} C_{1}^{x 2}=0 e$ and $q^{y}=e N^{y} C_{1}^{y 2}=16 e$. For the triple- $Q$ spin texture, the currents over ten periods are shown in Fig. 5(c) that gives $q^{x}=0.00 e$ and $q^{y}=31.97 e$. The transported charges are $q^{x}=e N^{x} C_{1}^{x 1}-e N^{x} C_{1}^{x 2}=0 e$ and $q^{y}=e N^{y} C_{1}^{y 1}-e N^{y} C_{1}^{y 2}=32 e$ according to Eq. (5). Apparently, the numerical results show a very good agreement with Eq. (5).

To trigger the nonlinear response, one needs to deform the spin texture. For the double- $Q$ spin texture, we apply a
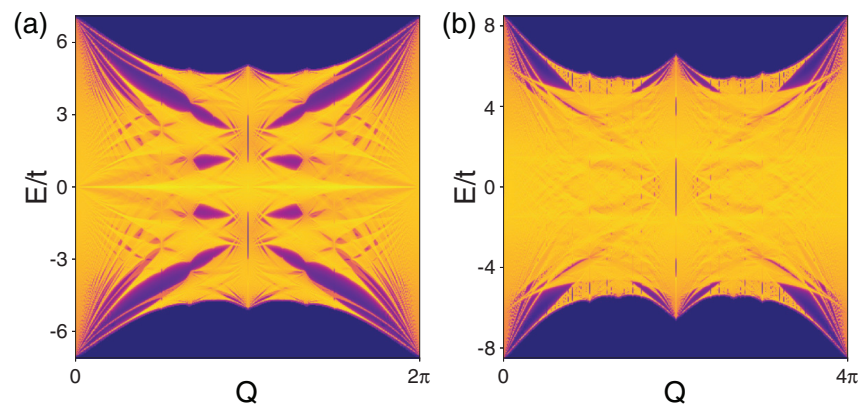

FIG. 6. Electronic spectral density as a function of $Q$ for the magnets with (a) the double- $Q$ collinear spin texture and (b) the triple- $Q$ collinear spin texture.

shear deformation of the spin texture by deforming the $\boldsymbol{Q}_{1}$ vector to $\boldsymbol{Q}_{1}=(2 \pi / 5,2 \pi / 20)$. By shifting the spin texture in the same way mentioned above, we obtain $q^{x}=-4.00 e$ and $q^{y}=16.00 e$ from the currents in Fig. 6(b). The numerical results are also consistent with $q^{x}=e L^{x} C_{2}^{x 21 y} Q_{1 y}^{\prime} / 2 \pi=-4 e$ and $q^{y}=16 e$ according to Eq. (10). For the triple- $Q$ spin texture, we apply a shear deformation of the spin texture by deforming the $\boldsymbol{Q}_{v}$ vectors to $\boldsymbol{Q}_{1}=(-2 \pi / 5,2 \sqrt{3} \pi / 5-$ $2 \sqrt{3} \pi / 20), \quad Q_{2}=(-2 \pi / 5,-2 \sqrt{3} \pi / 5-2 \sqrt{3} \pi / 20), \quad$ and $\boldsymbol{Q}_{3}=(4 \pi / 5,2 \sqrt{3} \pi / 10)$. In this case, the currents over ten periods are shown in Fig. 5(d) that gives $q^{x}=-7.98 e$ and $q^{y}=31.96 e$. Equation (5) yields $q^{x}=e L^{x} C_{2}^{x 12 y} Q_{2 y}^{\prime} / 2 \pi-$ $e L^{x} C_{2}^{x 21 y} Q_{1 y}^{\prime} / 2 \pi=-8 e$ and $q^{y}=32 e$ as before, which agree with the numerical results. The numerical results of anomalous charge transport for the spin texture ansatz are perfectly quantized and exhibit very good agreement with the response function.

To study how the electronic structure depends on the spin texture size, i.e., the $Q=\left|\boldsymbol{Q}_{v}\right|$, we show the spectral density as a function of $Q$ for the double- $Q$ and triple- $Q$ ansatz in Fig. 4. The spectra are reminiscent of the Hofstadter butterfly that exhibits topological energy gaps characterized by the nonzero Chern numbers and topological phase transitions induced by the change of magnetic field [58]. Because the $\boldsymbol{Q}_{v}$ vector plays the role as a magnetic field, the topological phase transition occurs through the change of $Q$ that is featured by the closing or opening of topological energy gaps.
[1] S. Mühlbauer, B. Binz, F. Jonietz, C. Pfleiderer, A. Rosch, A. Neubauer, R. Georgii, and P. Böni, Skyrmion lattice in a chiral magnet, Science 323, 915 (2009).

[2] X. Z. Yu, Y. Onose, N. Kanazawa, J. H. Park, J. H. Han, Y. Matsui, N. Nagaosa, and Y. Tokura, Real-space observation of a two-dimensional skyrmion crystal, Nature (London) 465, 901 (2010).

[3] S. Heinze, K. von Bergmann, M. Menzel, J. Brede, A. Kubetzka, R. Wiesendanger, G. Bihlmayer, and S. Blügel, Spontaneous atomic-scale magnetic skyrmion lattice in two dimensions, Nat. Phys. 7, 713 (2011).
[4] S. Seki, X. Z. Yu, S. Ishiwata, and Y. Tokura, Observation of skyrmions in a multiferroic material, Science 336, 198 (2012).

[5] F. Jonietz, S. Mühlbauer, C. Pfleiderer, A. Neubauer, W. Münzer, A. Bauer, T. Adams, R. Georgii, P. Böni, R. A. Duine, K. Everschor, M. Garst, and A. Rosch, Spin transfer torques in MnSi at ultralow current densities, Science 330, 1648 (2010).

[6] X. Z. Yu, N. Kanazawa, W. Z. Zhang, T. Nagai, T. Hara, K. Kimoto, Y. Matsui, Y. Onose, and Y. Tokura, Skyrmion flow near room temperature in an ultralow current density, Nat. Commun. 3, 988 (2012). 
[7] T. Schulz, R. Ritz, A. Bauer, M. Halder, M. Wagner, C. Franz, C. Pfleiderer, K. Everschor, M. Garst, and A. Rosch, Emergent electrodynamics of skyrmions in a chiral magnet, Nat. Phys. 8, 301 (2012).

[8] J. Zang, M. Mostovoy, J. H. Han, and N. Nagaosa, Dynamics of Skyrmion Crystals in Metallic Thin Films, Phys. Rev. Lett. 107, 136804 (2011).

[9] S.-Z. Lin, C. Reichhardt, C. D. Batista, and A. Saxena, Driven Skyrmions and Dynamical Transitions in Chiral Magnets, Phys. Rev. Lett. 110, 207202 (2013).

[10] J. Iwasaki, M. Mochizuki, and N. Nagaosa, Currentinduced skyrmion dynamics in constricted geometries, Nat. Nanotechnol. 8, 742 (2013).

[11] J. S. White, I. Levatic, A. A. Omrani, N. Egetenmeyer, K. Prsa, I. Zivkovic, J. L. Gavilano, J. Kohlbrecher, M. Bartkowiak, H. Berger, and H. M. Ronnow, Electric field control of the skyrmion lattice in $\mathrm{Cu}_{2} \mathrm{OSeO}_{3}$, J. Phys.: Condens. Matter 24, 432201 (2012).

[12] Y.-H. Liu, Y.-Q. Li, and J. H. Han, Skyrmion dynamics in multiferroic insulators, Phys. Rev. B 87, 100402(R) (2013).

[13] W. Wang, M. Beg, B. Zhang, W. Kuch, and H. Fangohr, Driving magnetic skyrmions with microwave fields, Phys. Rev. B 92, 020403(R) (2015).

[14] S. L. Zhang, W. W. Wang, D. M. Burn, H. Peng, H. Berger, A. Bauer, C. Pfleiderer, G. van der Laan, and T. Hesjedal, Manipulation of skyrmion motion by magnetic field gradients, Nat. Commun. 9, 2115 (2018).

[15] L. Kong and J. Zang, Dynamics of an Insulating Skyrmion Under a Temperature Gradient, Phys. Rev. Lett. 111, 067203 (2013).

[16] S.-Z. Lin, C. D. Batista, C. Reichhardt, and A. Saxena, Ac Current Generation in Chiral Magnetic Insulators and Skyrmion Motion Induced by the Spin Seebeck Effect, Phys. Rev. Lett. 112, 187203 (2014)

[17] Y. Onose, T. Ideue, H. Katsura, Y. Shiomi, N. Nagaosa, and Y. Tokura, Observation of the magnon Hall effect, Science 329, 297 (2010).

[18] A. Chacon, A. Bauer, T. Adams, F. Rucker, G. Brandl, R. Georgii, M. Garst, and C. Pfleiderer, Uniaxial Pressure Dependence of Magnetic Order in Mnsi, Phys. Rev. Lett. 115, 267202 (2015).

[19] Y. Nii, T. Nakajima, A. Kikkawa, Y. Yamasaki, K. Ohishi, J. Suzuki, Y. Taguchi, T. Arima, Y. Tokura, and Y. Iwasa, Uniaxial stress control of skyrmion phase, Nat. Commun. 6, 8539 (2015).

[20] K. Shibata, J. Iwasaki, N. Kanazawa, S. Aizawa, T. Tanigaki, M. Shirai, T. Nakajima, M. Kubota, M. Kawasaki, H. S. Park, D. Shindo, N. Nagaosa, and Y. Tokura, Large anisotropic deformation of skyrmions in strained crystal, Nat. Nanotechnol. 10, 589 (2015).

[21] A. Fert, V. Cros, and J. Sampaio, Skyrmions on the track, Nat. Nanotechnol. 8, 152 (2013).

[22] N. Nagaosa and Y. Tokura, Topological properties and dynamics of magnetic skyrmions, Nat. Nanotechnol. 8, 899 (2013).

[23] R. Wiesendanger, Nanoscale magnetic skyrmions in metallic films and multilayers: A new twist for spintronics, Nat. Rev. Mater. 1, 16044 (2016).

[24] N. Nagaosa, J. Sinova, S. Onoda, A. H. MacDonald, and N. P. Ong, Anomalous Hall effect, Rev. Mod. Phys. 82, 1539 (2010).
[25] Y. Su and S.-Z. Lin, Nontrivial topology and localization in the double exchange model with possible applications to perovskite manganites, Phys. Rev. B 98, 235116 (2018).

[26] A. Neubauer, C. Pfleiderer, B. Binz, A. Rosch, R. Ritz, P. G. Niklowitz, and P. Böni, Topological Hall Effect in the a Phase of MnSi, Phys. Rev. Lett. 102, 186602 (2009).

[27] Y. Li, N. Kanazawa, X. Z. Yu, A. Tsukazaki, M. Kawasaki, M. Ichikawa, X. F. Jin, F. Kagawa, and Y. Tokura, Robust Formation of Skyrmions and Topological Hall Effect Anomaly in Epitaxial Thin Films of Mnsi, Phys. Rev. Lett. 110, 117202 (2013).

[28] C. Franz, F. Freimuth, A. Bauer, R. Ritz, C. Schnarr, C. Duvinage, T. Adams, S. Blügel, A. Rosch, Y. Mokrousov, and C. Pfleiderer, Real-Space and Reciprocal-Space Berry Phases in the Hall Effect of $\mathrm{Mn}_{1-x} \mathrm{Fe}_{x} \mathrm{Si}$, Phys. Rev. Lett. 112, 186601 (2014).

[29] I. Martin and C. D. Batista, Itinerant Electron-Driven Chiral Magnetic Ordering and Spontaneous Quantum Hall Effect in Triangular Lattice Models, Phys. Rev. Lett. 101, 156402 (2008).

[30] C. D. Batista, S.-Z. Lin, S. Hayami, and Y. Kamiya, Frustration and chiral orderings in correlated electron systems, Rep. Prog. Phys. 79, 084504 (2016).

[31] S. Sorn, S. Divic, and A. Paramekanti, Tunable skyrmion crystals and topological quantum oscillations in magnetic metals, Phys. Rev. B 100, 174411 (2019).

[32] S. E. Barnes and S. Maekawa, Generalization of Faraday's Law to Include Nonconservative Spin Forces, Phys. Rev. Lett. 98, 246601 (2007).

[33] S. A. Yang, G. S. D. Beach, C. Knutson, D. Xiao, Q. Niu, M. Tsoi, and J. L. Erskine, Universal Electromotive Force Induced by Domain Wall Motion, Phys. Rev. Lett. 102, 067201 (2009).

[34] K. M. D. Hals and A. Brataas, Spin-motive forces and currentinduced torques in ferromagnets, Phys. Rev. B 91, 214401 (2015).

[35] J. Iwasaki, M. Mochizuki, and N. Nagaosa, Universal currentvelocity relation of skyrmion motion in chiral magnets, Nat. Commun. 4, 1463 (2013).

[36] D. Xiao, M.-C. Chang, and Q. Niu, Berry phase effects on electronic properties, Rev. Mod. Phys. 82, 1959 (2010).

[37] C. N. Yang, Generalization of dirac's monopole to $\mathrm{Su}_{2}$ gauge fields, J. Math. Phys. 19, 320 (1978).

[38] T. Fukui, Y. Hatsugai, and H. Suzuki, Chern numbers in discretized Brillouin zone: Efficient method of computing (spin) Hall conductances, J. Phys. Soc. Jpn. 74, 1674 (2005).

[39] M. Mochol-Grzelak, A. Dauphin, A. Celi, and M. Lewenstein, Efficient algorithm to compute the second chern number in four dimensional systems, Quantum Sci. Technol. 4, 014009 (2018).

[40] D. N. Sheng, L. Sheng, Z. Y. Weng, and F. D. M. Haldane, Spin Hall effect and spin transfer in a disordered Rashba model, Phys. Rev. B 72, 153307 (2005).

[41] J. S. White, K. Prša, P. Huang, A. A. Omrani, I. Živković, M. Bartkowiak, H. Berger, A. Magrez, J. L. Gavilano, G. Nagy, J. Zang, and H. M. Rønnow, Electric-Field-Induced Skyrmion Distortion and Giant Lattice Rotation in the Magnetoelectric Insulator $\mathrm{Cu}_{2} \mathrm{OSeO}_{3}$, Phys. Rev. Lett. 113, 107203 (2014).

[42] D. Okuyama, M. Bleuel, J. S. White, Q. Ye, J. Krzywon, G. Nagy, Z. Q. Im, I. Živković, M. Bartkowiak, H. M. Rønnow et al., Deformation of the moving magnetic skyrmion lattice in mnsi under electric current flow, Commun. Phys. 2, 79 (2019). 
[43] A. Garnier, D. Gignoux, B. Ouladdiaf, D. Schmitt, and T. Shigeoka, Magnetic incommensurability in tetragonal DyRu $\mathrm{Ge}_{2}$, Physica B: Condens. Matter 254, 166 (1998).

[44] G. G. Marcus, D.-J. Kim, J. A. Tutmaher, J. A. RodriguezRivera, J. O. Birk, C. Niedermeyer, H. Lee, Z. Fisk, and C. L. Broholm, Multi- $q$ Mesoscale Magnetism in $\mathrm{CeAuSb}_{2}$, Phys. Rev. Lett. 120, 097201 (2018).

[45] W. Jiang, P. Upadhyaya, W. Zhang, G. Yu, M. B. Jungfleisch, F. Y. Fradin, J. E. Pearson, Y. Tserkovnyak, K. L. Wang, O. Heinonen, S. G. E. te Velthuis, and A. Hoffmann, Blowing magnetic skyrmion bubbles, Science 349, 283 (2015).

[46] W. Jiang, X. Zhang, G. Yu, W. Zhang, X. Wang, M. Benjamin Jungfleisch, J. E. Pearson, X. Cheng, O. Heinonen, K. L. Wang, Y. Zhou, A. Hoffmann, and S. G. E. te Velthuis, Direct observation of the skyrmion Hall effect, Nat. Phys. 13, 162 (2017).

[47] K. Litzius, I. Lemesh, B. Krüger, P. Bassirian, L. Caretta, K. Richter, F. Büttner, K. Sato, O. A. Tretiakov, J. Förster, R. M. Reeve, M. Weigand, I. Bykova, H. Stoll, G. Schütz, G. S. D. Beach, and M. Kläui, Skyrmion Hall effect revealed by direct time-resolved X-ray microscopy, Nat. Phys. 13, 170 (2017).

[48] Q. Shao, Y. Liu, G. Yu, S. K. Kim, X. Che, C. Tang, Q. L. He, Y. Tserkovnyak, J. Shi, and K. L. Wang, Topological Hall effect at above room temperature in heterostructures composed of a magnetic insulator and a heavy metal, Nat. Electron. 2, 182 (2019).

[49] S. Ding, A. Ross, R. Lebrun, S. Becker, K. Lee, I. Boventer, S. Das, Y. Kurokawa, S. Gupta, J. Yang et al., Interfacial Dzyaloshinskii-Moriya interaction and chiral magnetic textures in a ferrimagnetic insulator, Phys. Rev. B 100, 100406 (2019).
[50] X. Li, W. V. Liu, and L. Balents, Spirals and Skyrmions in Two Dimensional Oxide Heterostructures, Phys. Rev. Lett. 112, 067202 (2014).

[51] D. Xiao, J. Shi, and Q. Niu, Berry Phase Correction to Electron Density of States in Solids, Phys. Rev. Lett. 95, 137204 (2005).

[52] H. M. Price, O. Zilberberg, T. Ozawa, I. Carusotto, and N. Goldman, Four-Dimensional Quantum Hall Effect with Ultracold Atoms, Phys. Rev. Lett. 115, 195303 (2015).

[53] Y. Gao, S. A. Yang, and Q. Niu, Field Induced Positional Shift of Bloch Electrons and its Dynamical Implications, Phys. Rev. Lett. 112, 166601 (2014).

[54] S. Weinberg, Gravitation and Cosmology: Principles and Applications of the General Theory of Relativity (Wiley, New York, 1972).

[55] S. Hayami, R. Ozawa, and Y. Motome, Effective bilinearbiquadratic model for noncoplanar ordering in itinerant magnets, Phys. Rev. B 95, 224424 (2017).

[56] R. Takagi, J. S. White, S. Hayami, R. Arita, D. Honecker, H. M. Rønnow, Y. Tokura, and S. Seki, Multiple-q noncollinear magnetism in an itinerant hexagonal magnet, Sci. Adv. 4, eaau3402 (2018).

[57] S. Hayami, S.-Z. Lin, and C. D. Batista, Bubble and skyrmion crystals in frustrated magnets with easy-axis anisotropy, Phys. Rev. B 93, 184413 (2016).

[58] D. R. Hofstadter, Energy levels and wave functions of Bloch electrons in rational and irrational magnetic fields, Phys. Rev. B 14, 2239 (1976).

[59] Y. E. Kraus, Z. Ringel, and O. Zilberberg, Four-Dimensional Quantum Hall Effect in a Two-Dimensional Quasicrystal, Phys. Rev. Lett. 111, 226401 (2013). 